\title{
Exact solution for buckling of axially-compressed cylindrical panels with frames attached to the circular edges
}

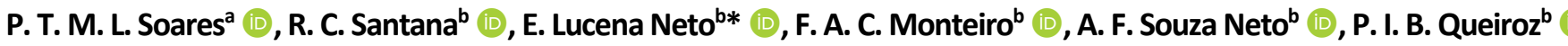 \\ ${ }^{a}$ Centro de Estudos e Projetos de Engenharia da Aeronáutica, 01552-000 São Paulo, SP, Brasil. E-mail: paulotsoares@gmail.com \\ b Instituto Tecnológico de Aeronáutica, 12228-900 São José dos Campos, SP, Brasil. E-mail: rafael.feg@gmail.com, eliseu@ita.br, \\ facm@ita.br, iei.Ime01@yahoo.com, pi@ita.br \\ * Corresponding author
}

https://doi.org/10.1590/1679-78255903

\begin{abstract}
This paper presents an exact solution for the boundary-value problem which describes the linear buckling of axially-compressed cylindrical panels with frames attached to the circular edges. The boundary conditions differ from the classical simply supported ones, often assumed for design purposes, in the sense that the torsion resisted by the frames are also taken into account. The quality of the results reported herein may be valuable benchmark data.
\end{abstract}

\section{Keywords}

Cylindrical panel, Buckling, Exact solution, Frame

\section{Graphical Abstract}
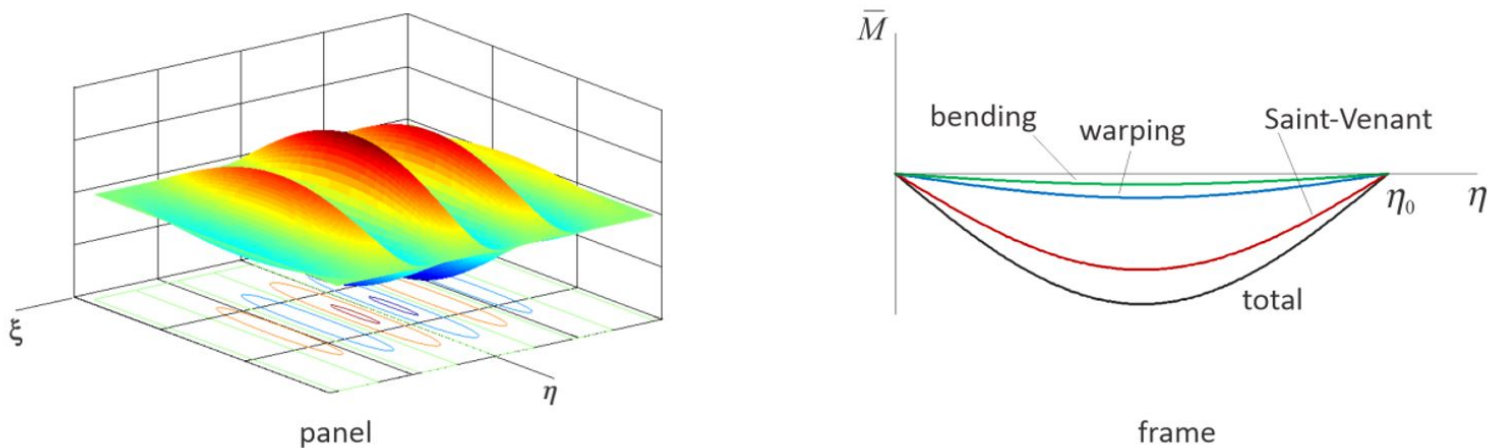

frame 


\section{INTRODUCTION}

A reinforced circular cylindrical shell is sketched in Figure 1, with skin, stringers (longitudinal stiffeners) and frames (circumferential stiffeners) displayed. An isolated panel, extracted from a skin portion between adjacent stiffeners, is also indicated. In order to better exploit the structure load capacity, as done for instance in a typical aeronautical design, the skin is assumed to buckle first (Kollár and Dulácska, 1984; Buermann et al., 2006). In this case, the isolated panel can thus be used as a simple solution domain to estimate the skin buckling.

The exact solution for buckling of axially-compressed cylindrical panels with all edges simply supported in the classical manner is easy to be found (Timoshenko and Gere, 1961). Chu and Krishnamoorthy (1967) reported an exact solution for the buckling of axially-compressed cylindrical panels with the circular edges simply supported and the straight edges free. By constraining the above free edges with stiffeners acting like beams, Krishnamoorthy (1974) proposed the first exact solution for panels with edge stiffeners.

More recently, axially compressed cylindrical panels with three edges simply supported and one edge free were treated by Magnucki and Maćkiewicz (2006) and by Wilde et al. (2007) using, respectively, the Galerkin and Lévy-type procedures. Varying the load from pure compression to full in-plane bending, the buckling of simply supported cylindrical panels was numerically analyzed by Martins et al. (2013). Parametric results for the buckling of axially-compressed cylindrically panels with the circular edges simply supported and the straight edges free were provided by

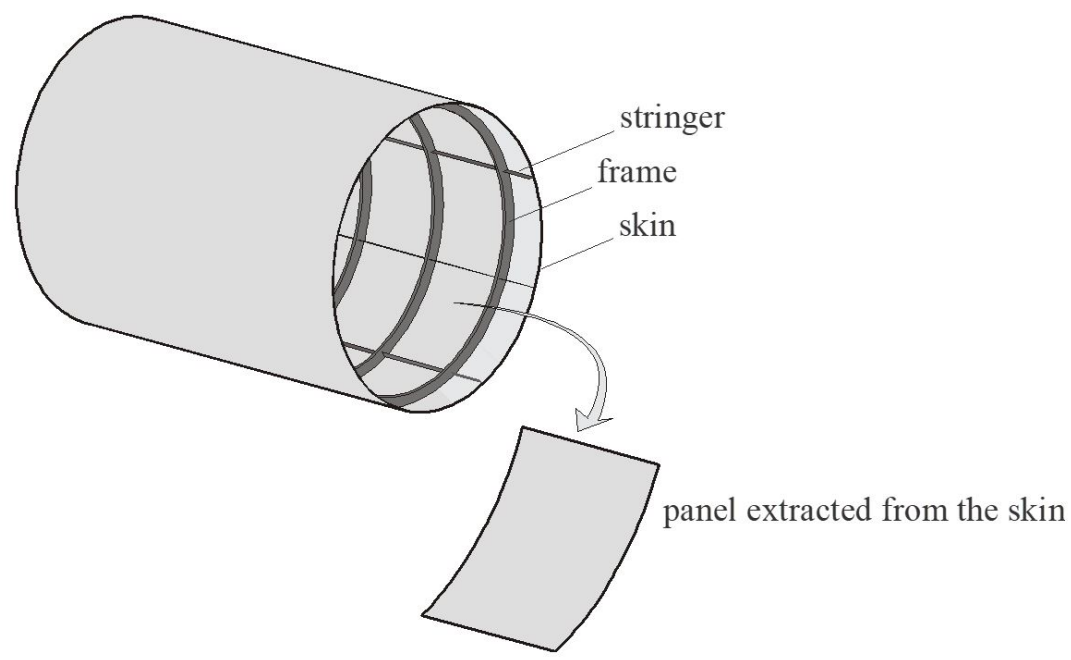

Figure 1: Cylindrical shell reinforced with stringers and frames.

Eipakchi and Shariati (2011), and by Afkhami et al. (2015) using, respectively, a perturbation technique and the Galerkin method.

Focusing on bridge construction aspects, Tran et al. (2012) studied the linear buckling and the ultimate strength of stiffened cylindrical panels under axial compression. Finally, the state-of-the-art on the stability behavior and design of cylindrical panels under generalized in-plane loading is well detailed in Martins et al. (2018). None of the previous works have treated the problem addressed here.

Axially-loaded stiffened cylindrical shells are generally low sensitive, or even insensitive, to imperfections depending on how closely stiffened they are (Koiter, 1956; Stephens, 1971; Singer, 2004). Due to the difficult of determining the actual imperfection, their design at present is still based on classical critical loads corrected by knockdown factors. In particular, with regard to the aerospace structures, prediction of knockdown factors remains highly dependent on the empirical guideline provided in NASA SP-8007 (Peterson et al., 1968). Moreover, the linearized buckling analysis can still be used to predict lower bounds on the critical load, as proposed by Sosa et al. (2006).

Following closely the work of Lucena Neto et al. (2016), one develops herein a procedure that exactly predict the linear buckling load for axially-compressed cylindrical panels with frames attached to the circular edges, identifying the external moments exerted by them on the buckling onset. From a mathematical point of view, the simple change of the stiffener from the straight edges (see Lucena Neto et al., 2016) to the circular ones in the panel is enough to make the problem challenging in stating the moments exerted by the frames on the circular edges and in establishing all the function spaces where a buckling mode might be. In the proposed solution, the frames are supposed to resist torsion in three ways: the first and second are denoted by Saint-Venant and warping torsions (Kollbrunner and Basler, 1969; Pilkey, 2002), respectively, while the third is associated with the frame out-of-plane bending. 
The linearized Donnell's equations, with prebuckling rotations neglected, are chosen to describe the panel buckling behavior (Brush and Almroth, 1975). The exact solution is stated here in a suitable detail, which may be valuable benchmark data.

\section{PROBLEM FORMULATION}

The circular cylindrical panel shown in Figure 2(a) has radius $R$, length $a$, width $b$ and thickness $h$. It is subjected to a uniformly distributed axial compressive force $p$ per unit length and referred to a set of orthogonal curvilinear coordinates $x y z$ placed in the panel midsurface. The panel buckling is supposed to be described by the linearized Donnell's equations (Brush and Almroth, 1975; Lucena Neto et al., 2016)

$$
\nabla^{4} u=-v w_{, \xi \xi \xi}+w_{, \xi \eta \eta} \quad \nabla^{4} v=-(2+v) w_{, \xi \xi \eta}-w_{, \eta \eta \eta} \quad \nabla^{8} w+w_{, \xi \xi \xi \xi}+2 \rho \nabla^{4} w_{, \xi \xi}=0
$$

with prebuckling rotations neglected. The nondimensional coordinates and displacements are defined as

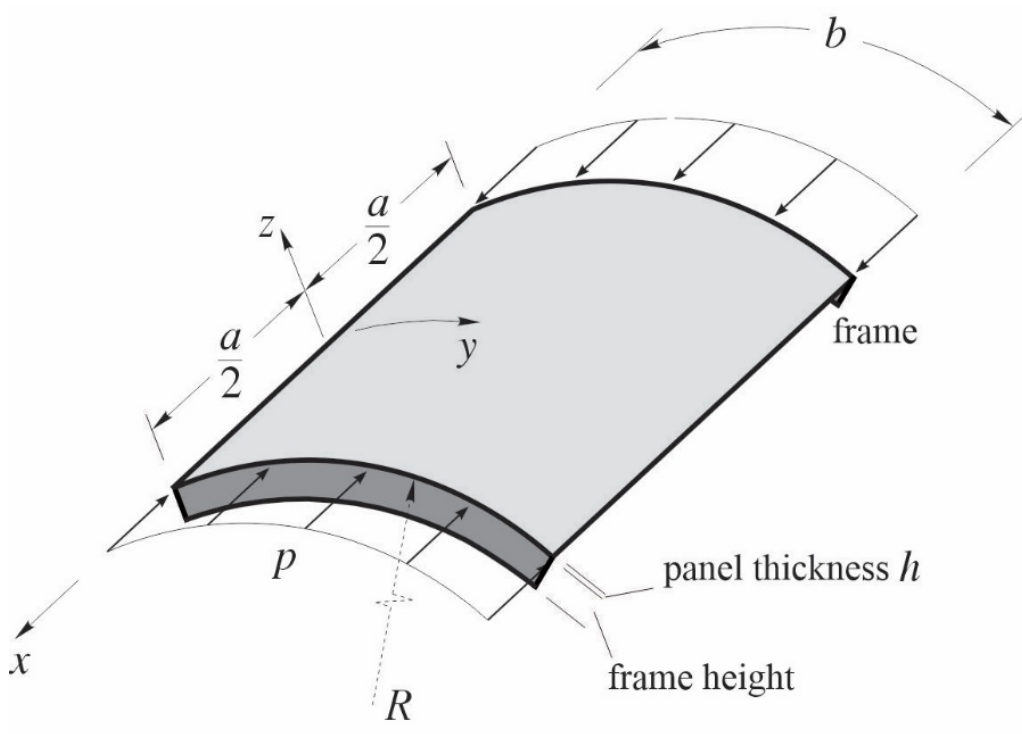

(a)

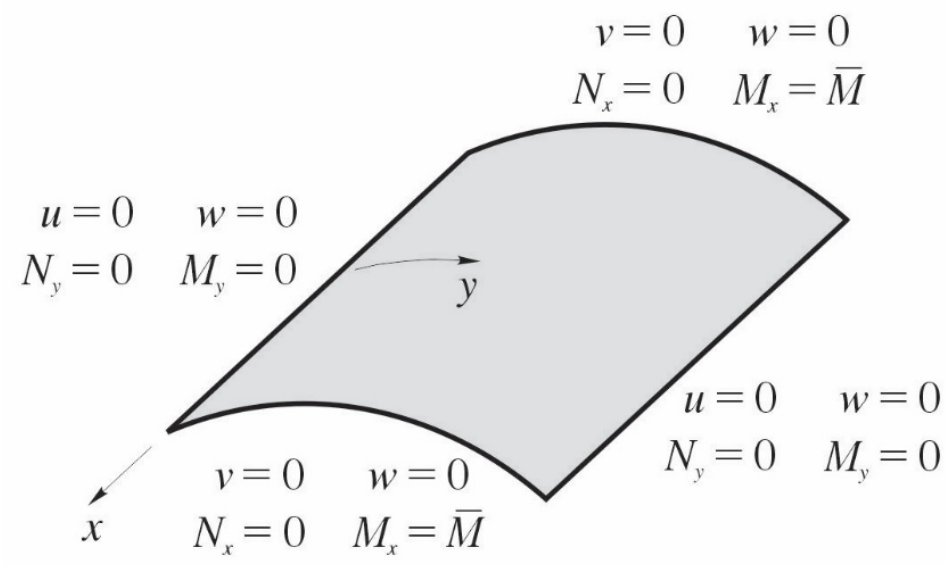

(b)

Figure 2: Cylindrical panel with frames attached to the circular edges: (a) geometry and loading; (b) boundary conditions. 


$$
\xi=\sqrt{\frac{2 E h}{p_{c l}}} \frac{x}{R} \quad \eta=\sqrt{\frac{2 E h}{p_{c l}}} \frac{y}{R} \quad u(\xi, \eta)=\sqrt{\frac{2 E h}{p_{c l}}} \frac{u(x, y)}{R} \quad v(\xi, \eta)=\sqrt{\frac{2 E h}{p_{c l}}} \frac{v(x, y)}{R} \quad w(\xi, \eta)=\frac{w(x, y)}{R}
$$

where

$$
p_{c l}=\frac{E h^{2}}{R \sqrt{3\left(1-v^{2}\right)}} \quad \rho=\frac{p}{p_{c l}} .
$$

The classical value $p_{c l}$ represents the minimum buckling load $p$ that a simply supported panel could ever achieve. Moreover, $u, v$ and $w$ are the midsurface displacements in the $x, y$ and $z$ directions; $E$ is the Young's modulus, $v$ is the Poisson's ratio, $\nabla^{8}$ denotes two successive applications of the two-dimensional biharmonic operator $\nabla^{4}(\quad)=()_{, \xi \xi \xi \xi}+2()_{, \xi \xi \eta \eta}+()_{, \eta \eta \eta \eta}$ and a comma followed by $\xi$ (or $\eta$ ) indicates differentiation with respect to $\xi$ (or $\eta$ ).

The boundary conditions (see Figure 2(b)) to be applied differ from the classical simply supported ones in the sense that the torsion resisted by the frames attached to the panel circular edges are also taken into account:

$$
\begin{array}{rrrrr}
N_{\xi}=0 & v=0 & w=0 & M_{\xi}=\bar{M} & \text { at } \xi= \pm \xi_{0} \\
u=0 & N_{\eta}=0 & w=0 & M_{\eta}=0 & \text { at } \eta=0, \eta_{0}
\end{array}
$$

with

$$
\xi_{0}=\sqrt{\frac{2 E h}{p_{c l}}} \frac{a}{2 R} \quad \eta_{0}=\sqrt{\frac{2 E h}{p_{c l}}} \frac{b}{R}
$$

The nondimensional in-plane forces $N_{\xi}$ and $N_{\eta}$, bending moments $M_{\xi}$ and $M_{\eta}$ and external moments $\bar{M}$ exerted by the frames on the panel circular edges $\xi= \pm \xi_{0}$ are

$$
\begin{aligned}
N_{\xi}= & \frac{E h}{1-v^{2}}\left(u_{, \xi}+v v_{, \eta}+v w\right) \quad N_{\eta}=\frac{E h}{1-v^{2}}\left(v u_{, \xi}+v_{, \eta}+w\right) \\
M_{\xi}= & -\frac{D}{R} \frac{2 E h}{p_{c l}}\left(w_{, \xi \xi}+v w_{, \eta \eta}\right) \quad M_{\eta}=-\frac{D}{R} \frac{2 E h}{p_{c l}}\left(v w_{, \xi \xi}+w_{, \eta \eta}\right) \\
\bar{M}\left( \pm \xi_{0}, \eta\right)= & \mp \frac{E_{f} I_{f}}{R^{2}} \sqrt{\frac{2 E h}{p_{c l}}}\left[u_{, \eta \eta}\left( \pm \xi_{0}, \eta\right)-w_{, \xi}\left( \pm \xi_{0}, \eta\right)\right] \mp \frac{G_{f} J_{f}}{R^{2}} \sqrt{\frac{2 E h}{p_{c l}}}\left[u_{, \eta \eta}\left( \pm \xi_{0}, \eta\right)+\frac{2 E h}{p_{c l}} w_{, \xi \eta \eta}\left( \pm \xi_{0}, \eta\right)\right] \\
& \pm \frac{E_{f} \Gamma_{f}}{R^{4}}\left(\frac{2 E h}{p_{c l}}\right)^{3 / 2}\left[u_{, \eta \eta \eta \eta}\left( \pm \xi_{0}, \eta\right)+\frac{2 E h}{p_{c l}} w_{, \xi \eta \eta \eta \eta}\left( \pm \xi_{0}, \eta\right)\right]
\end{aligned}
$$

where $D=E h^{3} / 12\left(1-v^{2}\right)$ is the panel bending rigidity.

Note that the external moment, which prevent the panel circular edges from rotating freely, is prescribed but not known until the problem is completed solved. Details on its expression development is given in Appendix $A$. The frames have torsional and warping constants $J_{f}$ and $\Gamma_{f}$, area moment of inertia of the cross section about the $z$ axis given by $I_{f}$, material with Young's modulus $E_{f}$ and shear modulus $G_{f}$. The first term in $\bar{M}$ is associated with the frame out-of-plane bending, while the second and third terms are associated with Saint-Venant and warping torsions, respectively. 


\section{CHARACTERISTIC EQUATION AND SOLUTION PROCEDURE}

A general buckling mode satisfying the simply supported boundary conditions on edges $\eta=0, \eta_{0}$, can be assumed in the form

$$
u(\xi, \eta)=U(\xi) \sin k \eta \quad v(\xi, \eta)=V(\xi) \cos k \eta \quad w(\xi, \eta)=W(\xi) \sin k \eta
$$

with

$$
k=\frac{n \pi R}{b} \sqrt{\frac{p_{c l}}{2 E h}}
$$

and the integer $n$ standing for the number of half-waves in the circumferential direction. The functions $U(\xi), V(\xi)$ and $W(\xi)$ must be obtained so that the mode fulfills the conditions required by the supports at $\xi= \pm \xi_{0}$ and satisfies (1).

After substitution of (7), the boundary conditions (4) on edges $\xi= \pm \xi_{0}$ hold for every $0<\eta<\eta_{0}$ if

$$
\begin{aligned}
& U_{, \xi}\left( \pm \xi_{0}\right)=0 \quad V\left( \pm \xi_{0}\right)=0 \quad W\left( \pm \xi_{0}\right)=0 \\
& \frac{D}{R} W_{, \xi \xi}\left( \pm \xi_{0}\right) \pm \sqrt{\frac{p_{c l}}{2 E h}}\left(\frac{E_{f} I_{f}}{R^{2}}+\frac{n^{2} \pi^{2}}{b^{2}} G_{f} J_{f}+\frac{n^{4} \pi^{4}}{b^{4}} E_{f} \Gamma_{f}\right) W_{, \xi}\left( \pm \xi_{0}\right) \\
& \pm \frac{n^{2} \pi^{2}}{b^{2}}\left(\frac{p_{c l}}{2 E h}\right)^{\frac{3}{2}}\left(E_{f} I_{f}+G_{f} J_{f}+\frac{n^{2} \pi^{2}}{b^{2}} E_{f} \Gamma_{f}\right) U\left( \pm \xi_{0}\right)=0 .
\end{aligned}
$$

On the other hand, the equation obtained after substitution of $w(\xi, \eta)$ into the third of equations (1) holds for every point $(\xi, \eta)$ of the domain for nontrivial $w$ (i.e., $W \neq 0$ ) if

$$
\frac{d^{8} W}{d \xi^{8}}-2\left(2 k^{2}-\rho\right) \frac{d^{6} W}{d \xi^{6}}+\left(6 k^{4}-4 \rho k^{2}+1\right) \frac{d^{4} W}{d \xi^{4}}-2 k^{4}\left(2 k^{2}-\rho\right) \frac{d^{2} W}{d \xi^{2}}+k^{8} W=0 .
$$

Particular solutions of this homogeneous linear differential equation are in the form $e^{s \xi}$, where $s$ denotes a root of the characteristic equation

$$
s^{8}-2\left(2 k^{2}-\rho\right) s^{6}+\left(6 k^{4}-4 \rho k^{2}+1\right) s^{4}-2 k^{4}\left(2 k^{2}-\rho\right) s^{2}+k^{8}=0 .
$$

Closed-form solutions of polynomial equations beyond sixth order are restricted, except in the numerical form (King, 1996;. McNamee, 2007; McNamee and Pan, 2013). Fortunately, equation (11) can be factored in the form

$$
\left(\frac{s^{2}-k^{2}}{s}\right)^{4}+2 \rho\left(\frac{s^{2}-k^{2}}{s}\right)^{2}+1=0
$$

regardless of the values of $k$ and $\rho$. Thus, its roots are

$$
s=\frac{ \pm i \sqrt{\lambda_{i}} \pm \sqrt{4 k^{2}-\lambda_{i}}}{2}
$$

where

$$
\lambda_{1}=\rho-\sqrt{\rho^{2}-1} \quad \lambda_{2}=\rho+\sqrt{\rho^{2}-1}
$$


and $i=\sqrt{-1}$ denotes the imaginary unit. It is clear from physical considerations that the buckling will always take place for $\rho>1$ due to the attachment of frames to the panel edges $\xi= \pm \xi_{0}$. In view of this evidence, the parameters $\lambda_{2}>\lambda_{1}>0$.

The presence of the constant term $k^{8}(k>0)$ in (11) and the property $\lambda_{i}>0$ anticipate that zero and real roots do not exist. Depending on the values of $\lambda_{i}$ and $k$, the roots may be grouped according to the five cases in the sequel.

3.1 Case I: $0<\boldsymbol{k}^{2}<\lambda_{1} / 4$

All the roots are distinct and purely imaginary:

$$
s_{1}=-s_{5}=i \gamma_{1} \quad s_{2}=-s_{6}=i \gamma_{2} \quad s_{3}=-s_{7}=i \gamma_{3} \quad s_{4}=-s_{8}=i \gamma_{4}
$$

with

$$
\left.\left.\begin{array}{l}
\gamma_{1} \\
\gamma_{2}
\end{array}\right\}=\frac{\sqrt{\lambda_{1}} \pm \sqrt{\lambda_{1}-4 k^{2}}}{2} \quad \begin{array}{l}
\gamma_{3} \\
\gamma_{4}
\end{array}\right\}=\frac{\sqrt{\lambda_{2}} \pm \sqrt{\lambda_{2}-4 k^{2}}}{2}
$$

The solution of (10) may then be taken in the form

$$
\begin{aligned}
W(\xi)= & W_{1} \sin \gamma_{1} \xi+W_{2} \cos \gamma_{1} \xi+W_{3} \sin \gamma_{2} \xi+W_{4} \cos \gamma_{2} \xi \\
& +W_{5} \sin \gamma_{3} \xi+W_{6} \cos \gamma_{3} \xi+W_{7} \sin \gamma_{4} \xi+W_{8} \cos \gamma_{4} \xi
\end{aligned}
$$

where $W_{i}$ are arbitrary constants.

Because the $y z$ plane is a symmetry plane for the structure (see Figure 2(a)), the buckling modes can be separated into two distinct symmetry classes, which may be readily identified by the shape of $W(\xi)$. The modes may be classified by whether the displacement component $w$ is symmetric or antisymmetric with respect to the $y z$ plane. The displacement components $u$ and $v$ will then also have appropriate symmetries. This separation not only aids in identifying and classifying the buckling mode, but also reduces the eigenvalue problem to two distinct problems with smaller determinants to be evaluated. Using the subscripts " $s$ " and " $a$ " to refer to symmetric and antisymmetric parts, Eq. (17) is split into

$$
\begin{aligned}
& W_{s}(\xi)=W_{1 s} \cos \gamma_{1} \xi+W_{2 s} \cos \gamma_{2} \xi+W_{3 s} \cos \gamma_{3} \xi+W_{4 s} \cos \gamma_{4} \xi \\
& W_{a}(\xi)=W_{1 a} \sin \gamma_{1} \xi+W_{2 a} \sin \gamma_{2} \xi+W_{3 a} \sin \gamma_{3} \xi+W_{4 a} \sin \gamma_{4} \xi
\end{aligned}
$$

where $W_{i s}$ and $W_{i a}$ are appropriate redefinitions of $W_{i}$. From (1), (7) and (18), the functions $U(\xi)$ and $V(\xi)$ may also be split into

$$
\begin{aligned}
& U_{s}(\xi)=W_{1 s} u_{1 s} \sin \gamma_{1} \xi+W_{2 s} u_{2 s} \sin \gamma_{2} \xi+W_{3 s} u_{3 s} \sin \gamma_{3} \xi+W_{4 s} u_{4 s} \sin \gamma_{4} \xi \\
& U_{a}(\xi)=W_{1 a} u_{1 a} \cos \gamma_{1} \xi+W_{2 a} u_{2 a} \cos \gamma_{2} \xi+W_{3 a} u_{3 a} \cos \gamma_{3} \xi+W_{4 a} u_{4 a} \cos \gamma_{4} \xi \\
& V_{s}(\xi)=W_{1 s} v_{1 s} \cos \gamma_{1} \xi+W_{2 s} v_{2 s} \cos \gamma_{2} \xi+W_{3 s} v_{3 s} \cos \gamma_{3} \xi+W_{4 s} v_{4 s} \cos \gamma_{4} \xi \\
& V_{a}(\xi)=W_{1 a} v_{1 a} \sin \gamma_{1} \xi+W_{2 a} v_{2 a} \sin \gamma_{2} \xi+W_{3 a} v_{3 a} \sin \gamma_{3} \xi+W_{4 a} v_{4 a} \sin \gamma_{4} \xi
\end{aligned}
$$

The coefficients $u_{i s}, u_{i a}, v_{i s}$ and $v_{i a}$ are detailed in Appendix $\mathrm{B}$.

Introduction of the symmetric (or antisymmetric) displacement components (18) and (19) into (9) for $\xi=\xi_{0}$ yields the homogeneous system of equations

$$
\mathbf{K W}=\mathbf{0} \text {, }
$$


where the vector $\mathbf{W}$ collects $W_{i s}$ (or $W_{i a}$ ) and the matrix $\mathbf{K}$ is given in Appendix $\mathbf{B}$. Each root $\rho$ of the equation det $\mathbf{K}=0$ represents a buckling load.

Since similar expressions to (20) hold for the remaining cases, for which the matrix $\mathbf{K}$ is also listed in Appendix B, only the functions $U(\xi), V(\xi)$ and $W(\xi)$ associated with the solution will be summarized next.

\subsection{Case II: $\boldsymbol{k}^{2}=\lambda_{1} / 4$}

As the parameters $\gamma_{1}=\gamma_{2}$ in (16), the roots (15) reduces to

$$
s_{1}=s_{2}=-s_{5}=-s_{6}=i \gamma_{1} \quad s_{3}=-s_{7}=i \gamma_{3} \quad s_{4}=-s_{8}=i \gamma_{4}
$$

The solutions (18) and (19) must be modified to account for the repeated roots $s_{1}=s_{2}$ and $s_{5}=s_{6}$ :

$$
\begin{aligned}
& W_{s}(\xi)=W_{1 s} \cos \gamma_{1} \xi+W_{2 s} \xi \sin \gamma_{1} \xi+W_{3 s} \cos \gamma_{3} \xi+W_{4 s} \cos \gamma_{4} \xi \\
& W_{a}(\xi)=W_{1 a} \sin \gamma_{1} \xi+W_{2 a} \xi \cos \gamma_{1} \xi+W_{3 a} \sin \gamma_{3} \xi+W_{4 a} \sin \gamma_{4} \xi \\
& U_{s}(\xi)=\left(W_{1 s} u_{1 s}+W_{2 s} u_{2 s}\right) \sin \gamma_{1} \xi+W_{2 s} \bar{u}_{2 s} \xi \cos \gamma_{1} \xi+W_{3 s} u_{3 s} \sin \gamma_{3} \xi+W_{4 s} u_{4 s} \sin \gamma_{4} \xi \\
& U_{a}(\xi)=\left(W_{1 a} u_{1 a}+W_{2 a} u_{2 a}\right) \cos \gamma_{1} \xi+W_{2 a} \bar{u}_{2 a} \xi \sin \gamma_{1} \xi+W_{3 a} u_{3 a} \cos \gamma_{3} \xi+W_{4 a} u_{4 a} \cos \gamma_{4} \xi \\
& V_{s}(\xi)=\left(W_{1 s} v_{1 s}+W_{2 s} v_{2 s}\right) \cos \gamma_{1} \xi+W_{2 s} \bar{v}_{2 s} \xi \sin \gamma_{1} \xi+W_{3 s} v_{3 s} \cos \gamma_{3} \xi+W_{4 s} v_{4 s} \cos \gamma_{4} \xi \\
& V_{a}(\xi)=\left(W_{1 a} v_{1 a}+W_{2 a} v_{2 a}\right) \sin \gamma_{1} \xi+W_{2 a} \bar{v}_{2 a} \xi \cos \gamma_{1} \xi+W_{3 a} v_{3 a} \sin \gamma_{3} \xi+W_{4 a} v_{4 a} \sin \gamma_{4} \xi
\end{aligned}
$$

The coefficients $u_{i s}, \bar{u}_{i s}, u_{i a}, \bar{u}_{i a}, v_{i s}, \bar{v}_{i s}, v_{i a}, \bar{v}_{i a}$ are detailed in Appendix B.

3.3 Case III: $\lambda_{1} / 4<k^{2}<\lambda_{2} / 4$

All the roots are distinct. Roots $s_{1}, s_{2}, s_{5}, s_{6}$ are the complex roots

$$
\left.\begin{array}{l}
s_{1}=-s_{5} \\
s_{2}=-s_{6}
\end{array}\right\}=\alpha_{1} \pm i \beta_{1}
$$

with

$$
\alpha_{1}=\frac{\sqrt{4 k^{2}-\lambda_{1}}}{2} \quad \beta_{1}=\frac{\sqrt{\lambda_{1}}}{2}
$$

and roots $s_{3}, s_{4}, s_{7}, s_{8}$ are the purely imaginary roots identified in Case I. Splitting the solution of (10) into symmetric and antisymmetric parts as before,

$$
\begin{aligned}
W_{s}(\xi)= & W_{1 s} \sinh \alpha_{1} \xi \sin \beta_{1} \xi+W_{2 s} \cosh \alpha_{1} \xi \cos \beta_{1} \xi+W_{3 s} \cos \gamma_{3} \xi+W_{4 s} \cos \gamma_{4} \xi \\
W_{a}(\xi)= & W_{1 a} \sinh \alpha_{1} \xi \cos \beta_{1} \xi+W_{2 a} \cosh \alpha_{1} \xi \sin \beta_{1} \xi+W_{3 a} \sin \gamma_{3} \xi+W_{4 a} \sin \gamma_{4} \xi \\
U_{s}(\xi)= & \left(W_{1 s} u_{1 s}+W_{2 s} u_{2 s}\right) \sinh \alpha_{1} \xi \cos \beta_{1} \xi+\left(W_{1 s} \bar{u}_{1 s}+W_{2 s} \bar{u}_{2 s}\right) \cosh \alpha_{1} \xi \sin \beta_{1} \xi \\
& +W_{3 s} u_{3 s} \sin \gamma_{3} \xi+W_{4 s} u_{4 s} \sin \gamma_{4} \xi \\
U_{a}(\xi)= & \left(W_{1 a} u_{1 a}+W_{2 a} u_{2 a}\right) \sinh \alpha_{1} \xi \sin \beta_{1} \xi+\left(W_{1 a} \bar{u}_{1 a}+W_{2 a} \bar{u}_{2 a}\right) \cosh \alpha_{1} \xi \cos \beta_{1} \xi \\
& +W_{3 a} u_{3 a} \cos \gamma_{3} \xi+W_{4 a} u_{4 a} \cos \gamma_{4} \xi \\
V_{s}(\xi)= & \left(W_{1 s} v_{1 s}+W_{2 s} v_{2 s}\right) \sinh \alpha_{1} \xi \sin \beta_{1} \xi+\left(W_{1 s} \bar{v}_{1 s}+W_{2 s} \bar{v}_{2 s}\right) \cosh \alpha_{1} \xi \cos \beta_{1} \xi
\end{aligned}
$$




$$
\begin{aligned}
& +W_{3 s} v_{3 s} \cos \gamma_{3} \xi+W_{4 s} v_{4 s} \cos \gamma_{4} \xi \\
V_{a}(\xi)= & \left(W_{1 a} v_{1 a}+W_{2 a} v_{2 a}\right) \sinh \alpha_{1} \xi \cos \beta_{1} \xi+\left(W_{1 a} \bar{v}_{1 a}+W_{2 a} \bar{v}_{2 a}\right) \cosh \alpha_{1} \xi \sin \beta_{1} \xi \\
& +W_{3 a} v_{3 a} \sin \gamma_{3} \xi+W_{4 a} v_{4 a} \sin \gamma_{4} \xi
\end{aligned}
$$

The coefficients $u_{i s}, \bar{u}_{i s}, u_{i a}, \bar{u}_{i a}, v_{i s}, \bar{v}_{i s}, v_{i a}, \bar{v}_{i a}$ are detailed in Appendix B.

3.4 Case IV: $\boldsymbol{k}^{2}=\lambda_{2} / \mathbf{4}$

Roots $s_{1}, s_{2}, s_{5}, s_{6}$ are the complex roots identified in Case III, whereas $s_{3}, s_{4}, s_{7}, s_{8}$ reduce to the purely imaginary roots

$$
s_{3}=s_{4}=-s_{7}=-s_{8}=i \gamma_{3}
$$

In order to account for the repeated roots $s_{3}=s_{4}$ and $s_{7}=s_{8}$, the solution (25) must be modified to read

$$
\begin{aligned}
W_{s}(\xi)= & W_{1 s} \sinh \alpha_{1} \xi \sin \beta_{1} \xi+W_{2 s} \cosh \alpha_{1} \xi \cos \beta_{1} \xi+W_{3 s} \cos \gamma_{3} \xi+W_{4 s} \xi \sin \gamma_{3} \xi \\
W_{a}(\xi)= & W_{1 a} \sinh \alpha_{1} \xi \cos \beta_{1} \xi+W_{2 a} \cosh \alpha_{1} \xi \sin \beta_{1} \xi+W_{3 a} \sin \gamma_{3} \xi+W_{4 a} \xi \cos \gamma_{3} \xi \\
U_{s}(\xi)= & \left(W_{1 s} u_{1 s}+W_{2 s} u_{2 s}\right) \sinh \alpha_{1} \xi \cos \beta_{1} \xi+\left(W_{1 s} \bar{u}_{1 s}+W_{2 s} \bar{u}_{2 s}\right) \cosh \alpha_{1} \xi \sin \beta_{1} \xi \\
& +\left(W_{3 s} u_{3 s}+W_{4 s} u_{4 s}\right) \sin \gamma_{3} \xi+W_{4 s} \bar{u}_{4 s} \xi \cos \gamma_{3} \xi \\
U_{a}(\xi)= & \left(W_{1 a} u_{1 a}+W_{2 a} u_{2 a}\right) \sinh \alpha_{1} \xi \sin \beta_{1} \xi+\left(W_{1 a} \bar{u}_{1 a}+W_{2 a} \bar{u}_{2 a}\right) \cosh \alpha_{1} \xi \cos \beta_{1} \xi \\
& +\left(W_{3 a} u_{3 a}+W_{4 a} u_{4 a}\right) \cos \gamma_{3} \xi+W_{4 a} \bar{u}_{4 a} \xi \sin \gamma_{3} \xi \\
V_{s}(\xi)= & \left(W_{1 s} v_{1 s}+W_{2 s} v_{2 s}\right) \sinh \alpha_{1} \xi \sin \beta_{1} \xi+\left(W_{1 s} \bar{v}_{1 s}+W_{2 s} \bar{v}_{2 s}\right) \cosh \alpha_{1} \xi \cos \beta_{1} \xi \\
& +\left(W_{3 s} v_{3 s}+W_{4 s} v_{4 s}\right) \cos \gamma_{3} \xi+W_{4 s} \bar{v}_{4 s} \xi \sin \gamma_{3} \xi \\
V_{a}(\xi)= & \left(W_{1 a} v_{1 a}+W_{2 a} v_{2 a}\right) \sinh \alpha_{1} \xi \cos \beta_{1} \xi+\left(W_{1 a} \bar{v}_{1 a}+W_{2 a} \bar{v}_{2 a}\right) \cosh \alpha_{1} \xi \sin \beta_{1} \xi \\
& +\left(W_{3 a} v_{3 a}+W_{4 a} v_{4 a}\right) \sin \gamma_{3} \xi+W_{4 a} \bar{v}_{4 a} \xi \cos \gamma_{3} \xi .
\end{aligned}
$$

The coefficients $u_{i s}, \bar{u}_{i s}, u_{i a}, \bar{u}_{i a}, v_{i s}, \bar{v}_{i s}, v_{i a}, \bar{v}_{i a}$ are detailed in Appendix B.

\subsection{Case V: $\lambda_{2} / 4<k^{2}$}

All the roots are distinct and complex:

$$
\left.\left.\begin{array}{l}
s_{1}=-s_{5} \\
s_{2}=-s_{6}
\end{array}\right\}=\alpha_{1} \pm i \beta_{1} \quad \begin{array}{l}
s_{3}=-s_{7} \\
s_{4}=-s_{8}
\end{array}\right\}=\alpha_{2} \pm i \beta_{2}
$$

with

$$
\alpha_{i}=\frac{\sqrt{4 k^{2}-\lambda_{i}}}{2} \quad \beta_{i}=\frac{\sqrt{\lambda_{i}}}{2}
$$

The symmetric and antisymmetric solutions of (10) are 


$$
\begin{aligned}
W_{s}(\xi)= & W_{1 s} \sinh \alpha_{1} \xi \sin \beta_{1} \xi+W_{2 s} \cosh \alpha_{1} \xi \cos \beta_{1} \xi+W_{3 s} \sinh \alpha_{2} \xi \sin \beta_{2} \xi+W_{4 s} \cosh \alpha_{2} \xi \cos \beta_{2} \xi \\
W_{a}(\xi)= & W_{1 a} \sinh \alpha_{1} \xi \cos \beta_{1} \xi+W_{2 a} \cosh \alpha_{1} \xi \sin \beta_{1} \xi+W_{3 a} \sinh \alpha_{2} \xi \cos \beta_{2} \xi+W_{4 a} \cosh \alpha_{2} \xi \sin \beta_{2} \xi \\
U_{s}(\xi)= & \left(W_{1 s} u_{1 s}+W_{2 s} u_{2 s}\right) \sinh \alpha_{1} \xi \cos \beta_{1} \xi+\left(W_{1 s} \bar{u}_{1 s}+W_{2 s} \bar{u}_{2 s}\right) \cosh \alpha_{1} \xi \sin \beta_{1} \xi \\
& +\left(W_{3 s} u_{3 s}+W_{4 s} u_{4 s}\right) \sinh \alpha_{2} \xi \cos \beta_{2} \xi+\left(W_{3 s} \bar{u}_{3 s}+W_{4 s} \bar{u}_{4 s}\right) \cosh \alpha_{2} \xi \sin \beta_{2} \xi \\
U_{a}(\xi)= & \left(W_{1 a} u_{1 a}+W_{2 a} u_{2 a}\right) \sinh \alpha_{1} \xi \sin \beta_{1} \xi+\left(W_{1 a} \bar{u}_{1 a}+W_{2 a} \bar{u}_{2 a}\right) \cosh \alpha_{1} \xi \cos \beta_{1} \xi \\
& +\left(W_{3 a} u_{3 a}+W_{4 a} u_{4 a}\right) \sinh \alpha_{2} \xi \sin \beta_{2} \xi+\left(W_{3 a} \bar{u}_{3 a}+W_{4 a} \bar{u}_{4 a}\right) \cosh \alpha_{2} \xi \cos \beta_{2} \xi \\
V_{s}(\xi)= & \left(W_{1 s} v_{1 s}+W_{2 s} v_{2 s}\right) \sinh \alpha_{1} \xi \sin \beta_{1} \xi+\left(W_{1 s} \bar{v}_{1 s}+W_{2 s} \bar{v}_{2 s}\right) \cosh \alpha_{1} \xi \cos \beta_{1} \xi \\
& +\left(W_{3 s} v_{3 s}+W_{4 s} v_{4 s}\right) \sinh \alpha_{2} \xi \sin \beta_{2} \xi+\left(W_{3 s} \bar{v}_{3 s}+W_{4 s} \bar{v}_{4 s}\right) \cosh \alpha_{2} \xi \cos \beta_{2} \xi \\
V_{a}(\xi)= & \left(W_{1 a} v_{1 a}+W_{2 a} v_{2 a}\right) \sinh \alpha_{1} \xi \cos \beta_{1} \xi+\left(W_{1 a} \bar{v}_{1 a}+W_{2 a} \bar{v}_{2 a}\right) \cosh \alpha_{1} \xi \sin \beta_{1} \xi \\
& +\left(W_{3 a} v_{3 a}+W_{4 a} v_{4 a}\right) \sinh \alpha_{2} \xi \cos \beta_{2} \xi+\left(W_{3 a} \bar{v}_{3 a}+W_{4 a} \bar{v}_{4 a}\right) \cosh \alpha_{2} \xi \sin \beta_{2} \xi .
\end{aligned}
$$

The coefficients $u_{i s}, \bar{u}_{i s}, u_{i a}, \bar{u}_{i a}, v_{i s}, \bar{v}_{i s}, v_{i a}, \bar{v}_{i a}$ are detailed in Appendix $\mathrm{B}$.

\section{RESULTS}

The nonlinear eigenvalue problem (20) cannot be written in a form to allow the direct use of some standard software package to solve it. The following iteration steps are then employed to identify the smallest root $\rho$ of $\operatorname{det} \mathbf{K}=0$ for a given $n$ associated with a symmetric or antisymmetric mode:

1. evaluate $k$ from (8);

2. if $k^{2}<1 / 4$ then Case I, II or III applies.

Case I: The root lies in Case I if $\rho \in \mathrm{I}_{1}=(1, \alpha)$, with $\alpha=\left(16 k^{4}+1\right) / 8 k^{2}$. Search if the sign of det $\mathbf{K}$ changes in the interval $\mathrm{I}_{1}$. The search is made dividing the open interval in $10^{4}$ equal subintervals, evaluating det $\mathbf{K}$ progressively from $\rho=1+\varepsilon$ to $\alpha-\varepsilon$, with $\varepsilon=10^{-6}$. If the sign of the determinant changes, there is a root in that subinterval. Go to step 5. Otherwise, go to Case II.

Case II: If $\operatorname{det} \mathbf{K}=0$ with $\rho=\alpha$ save the root and stop the search for the given $n$. Otherwise, go to Case III. It has been accepted as null the value $\operatorname{det} \mathbf{K}<10^{-9}$.

Case III: The root lies in Case III if $\rho>\alpha$. Search if the sign of det $\mathbf{K}$ changes in the open interval $\mathrm{I}_{2}=(\alpha, 2 \alpha)$ similarly to Case I. If the sign changes, there is a root in that subinterval. Go to step 5. Otherwise, stop the search for the given $n$;

3. if $k^{2}=1 / 4$ then only Case III applies. Do Case III;

4. if $k^{2}>1 / 4$ then Case $V, I V$ or III applies.

Case $V$ : The root lies in Case $V$ if $\rho \in \mathrm{I}_{1}$. Search if the sign of det $\mathbf{K}$ changes similarly to Case $I$. If the sign changes, there is a root in that subinterval. Go to step 5. Otherwise, go to Case IV.

Case IV: If $\operatorname{det} \mathbf{K}=0$ with $\rho=\alpha$ save the root and stop the search for the given $n$. Otherwise, go to Case III.

Case III: do Case III;

5. search for the root in the subinterval where det $\mathbf{K}$ changes sign using some iterative methods. Herein one has employed the fzero function of MATLAB (2016) that finds roots of nonlinear functions using a combination of bisection, secant, and inverse quadratic interpolation methods. Save the root.

The critical value of $\rho$ corresponds to the smallest value obtained for $n=1,2,3, \ldots$ and for symmetric or antisymmetric modes. It is expected that the Cases $I /$ and $I V$ will be rarely activated. 
Table 1 Effect of the ratios $b / a$ and $R / h$, and frame support on the buckling load $\rho$.

\begin{tabular}{|c|c|c|c|c|}
\hline \multirow{2}{*}{$\left(U_{f}, \Gamma_{f}, I_{f}\right)$} & \multirow{2}{*}{$b / a$} & \multicolumn{3}{|c|}{$R / h$} \\
\hline & & 500 & 1000 & 1500 \\
\hline \multirow{10}{*}{$(0,0,0)$} & 0.2 & $1.00171(1, a, I)^{a}$ & $1.01779(1, \mathrm{~s}, \mathrm{IV})$ & $1.18156(1, \mathrm{~s}, \mathrm{IV})$ \\
\hline & 0.4 & $1.00152(1, a, l)$ & $1.00256(1, a, I)$ & $1.00122(1, \mathrm{~s}, \mathrm{I})$ \\
\hline & 0.6 & $1.00004(2, a, l)$ & $1.00102(1, \mathrm{~s}, \mathrm{l})$ & $1.00004(1, \mathrm{~s}, \mathrm{l})$ \\
\hline & 0.8 & $1.00000(5, a, l)^{b}$ & $1.00012(3, a, I)$ & $1.00026(3, a, I I I)$ \\
\hline & 1.0 & $1.00018(3, a, l)$ & $1.00000(1, \mathrm{~s}, \mathrm{l})^{\mathrm{b}}$ & $1.00017(3, a, l)$ \\
\hline & 1.2 & $1.00004(4, a, l)$ & $1.00001(1, \mathrm{~s}, \mathrm{l})$ & $1.00004(2, s, I)$ \\
\hline & 1.4 & $1.00000(9, a, I I I)^{b}$ & $1.00001(6, s, I)$ & $1.00018(4, a, l)$ \\
\hline & 0.2 & $1.00618(1, a, l)$ & $1.02644(1, \mathrm{a}, \mathrm{III})$ & $1.20792(1, \mathrm{~s}, \mathrm{III})$ \\
\hline & 0.4 & $1.00618(2, a, l)$ & $1.01775(1, a, I)$ & $1.00402(1, \mathrm{~s}, \mathrm{l})$ \\
\hline & 0.6 & $1.00430(4, a, I I I)$ & $1.01338(2, a, I)$ & $1.00295(2, a, l)$ \\
\hline \multirow[t]{4}{*}{$(96,36370,853)$} & 0.8 & $1.00047(5, s, I)$ & $1.00355(3, a, I)$ & $1.00363(3, s, I I I)$ \\
\hline & 1.0 & $1.00085(6, a, l)$ & $1.00578(4, \mathrm{~s}, \mathrm{l})$ & $1.01160(3, a, l)$ \\
\hline & 1.2 & $1.00230(7, a, l)$ & $1.00288(5, \mathrm{~s}, \mathrm{l})$ & $1.00295(4, a, l)$ \\
\hline & 1.4 & $1.00050(9, \mathrm{a}, \mathrm{III})$ & $1.00170(6, s, I)$ & $1.00085(5, \mathrm{~s}, \mathrm{l})$ \\
\hline
\end{tabular}

${ }^{a}$ Circumferential half-wave number, s: symmetric or a: antisymmetric mode, solution case. ${ }^{b}$ Slightly greater than unity.

All the analyzed panels have length $a=500 \mathrm{~mm}$, thickness $h=1 \mathrm{~mm}$, and material defined by $E=72400 \mathrm{~N} / \mathrm{mm}^{2}$, $v=0.33$. Several values are attributed to the width $b$ and radius $R$. The frame has $J_{f}=96 \mathrm{~mm}^{4}, \Gamma_{f}=36370 \mathrm{~mm}^{6}$ and $I_{f}=853 \mathrm{~mm}^{4}$ and material given by $E_{f}=71020 \mathrm{~N} / \mathrm{mm}^{2}$ and $G_{f}=26700 \mathrm{~N} / \mathrm{mm}^{2}$.

Table 1 summarizes the effect of the aspect ratio $b / a$ and radius-to-thickness ratio $R / h$ on the critical value of $\rho$. The half-wave number $(n)$, mode type (symmetric, antisymmetric), and solution case (I, II, III, IV, $\mathrm{V}$ ) are indicated in parentheses. The triple $U_{f}=0, \Gamma_{f}=0, I_{f}=0$ ) identifies panels under the classical simply supported boundary conditions. The half-wave number for panels with frames never decreases as $b / a$ increases (wide panels).

Figure 3 depicts the buckling modes for the following panels with frames: $R / h=1000$ and $b / a=0.4,0.6$ and 1.4 , including the moments exerted by the frame on the circular edge $\xi=\xi_{0}$. As expected, the warping torsion contribution becomes more significant for buckling modes with higher circumferential half-wave numbers. It is interesting to note that, depending on the membrane effect, the out-of-plane bending torsion may have opposite sign compared to that of the Saint-Venant and warping torsions.

The load parameter $p a^{2} / \pi^{2} D$ is plotted in Figure 4 against the curvature parameter $Z=\sqrt{1-v^{2}} a^{2} / R h$ on a $\log$ - log scale for panels with and without frames, and aspect ratios $b / a=0.5,1$ and 2 . The curves are obtained varying $R$ while all the remaining parameters are kept constant. The curves show that the influence of the frames is highly dependent on the curvature parameter. For instance, the frames may increase the buckling load as high as $37 \%$ for $Z=2.85$ and $b / a=1$ and decrease it as $Z$ increases. After $Z \approx 100$, the curves become nearly indistinguishable. The four marked points on each curve of Figure 4 represents the values of the critical load obtained from a Ritz procedure based on a complete hierarchic set of polynomial functions (Bardell, 1991; Yshii et al., 2018). The Ritz solutions are included here as a simple way of illustrating how the proposed results may be useful as benchmarks. The maximum difference between the exact and Ritz results is lower than $0.05 \%$, observed for the panel with frames and $b / a=0.5$ and $Z=100$. 

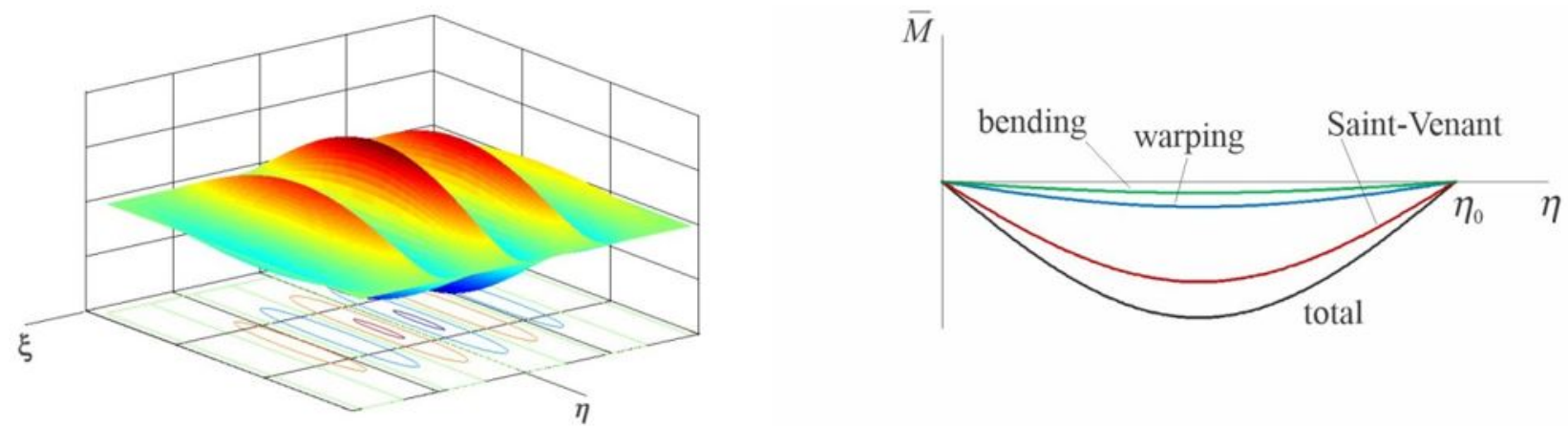

(a)
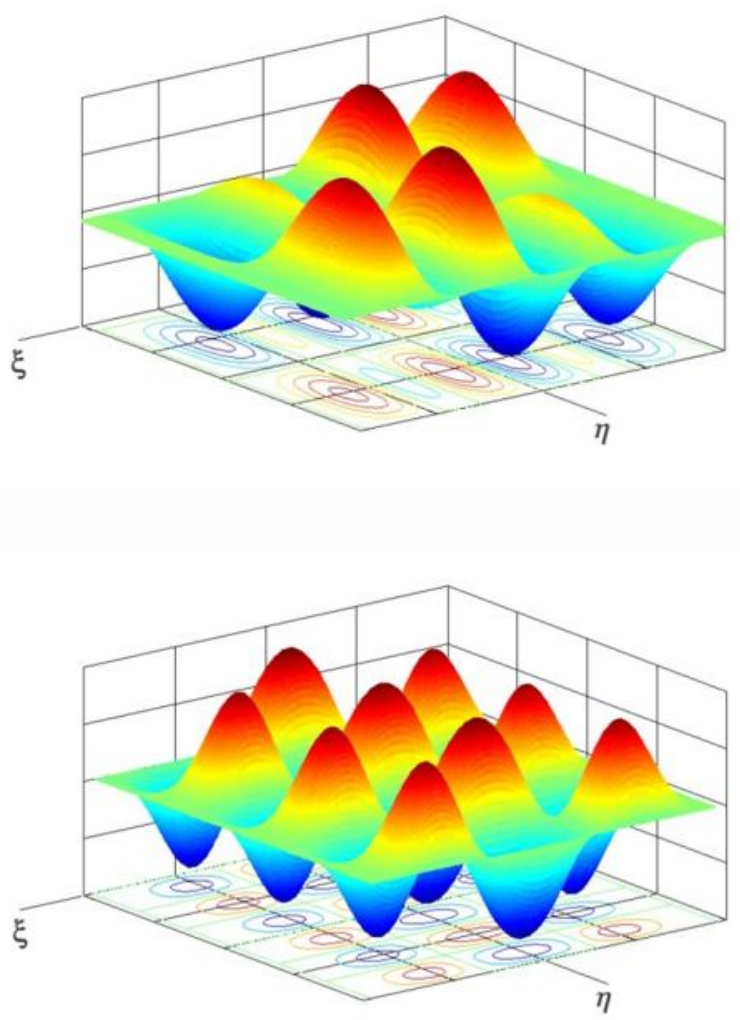

(b)
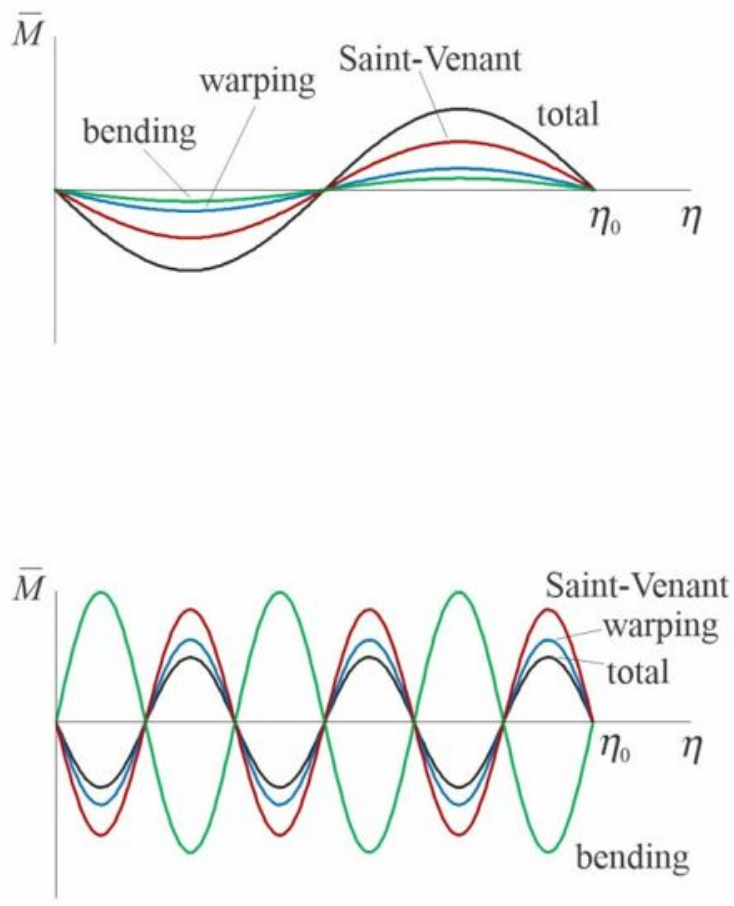

(c)

Figure 3: Buckling modes and external moments $\bar{M}$ exerted by the frame on the edges $\xi=\xi_{0}$ for panels with $R / h=1000:(a)$ $b / a=0.4$; (b) $b / a=0.6$; (c) $b / a=1.4$.

\section{CONCLUSION}

A Lévy-type procedure is adopted to develop, in a suitable detail, an exact solution for buckling of axiallycompressed cylindrical panels with frames attached to the circular edges. The linearized Donnell's equations, with prebuckling rotations neglected, are chosen to describe the buckling behavior. Both Saint-Venant and warping torsions, as well as the frame out-of-plane bending, are taken into account in the torsion resisted by the frames. The eight order characteristic equation is solved in closed form. An algorithm to generate numerical results is provided. Sets of exact results for specific panels and frames are tabled with which those from approximated procedures may be directly compared. While attached stringers on the straight edges may rise the buckling load to around $51 \%$ with respect to simply supported boundary conditions, as reported by Lucena Neto et al. (2016), the attachment of frames on the circular edges may rise the buckling load as high as $37 \%$ considering the set of analyzed panels. 


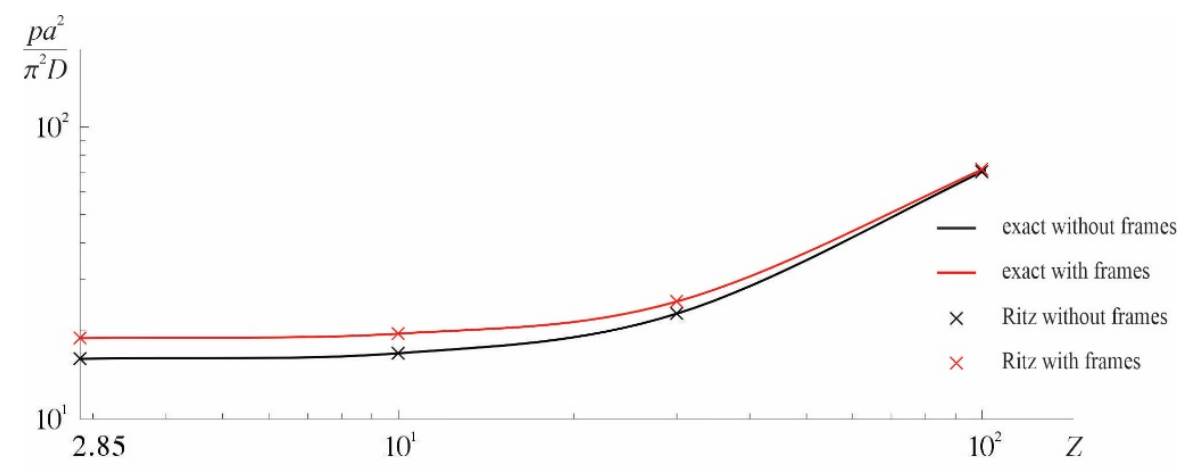

(a)

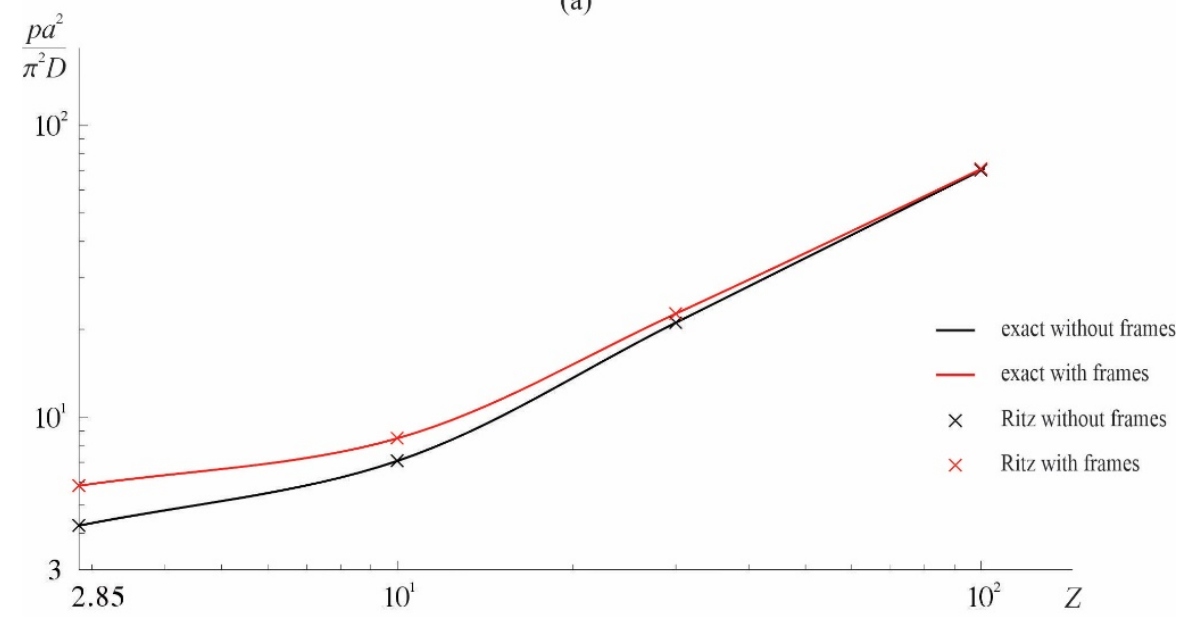

(b)

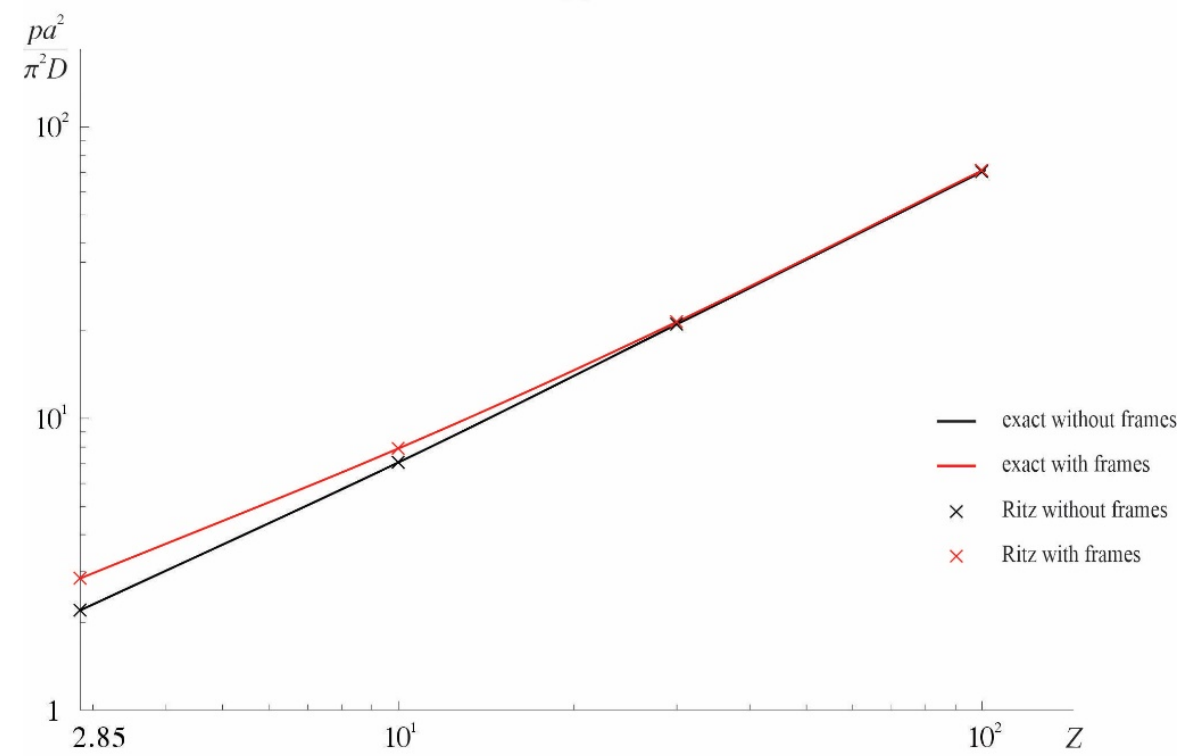

(c)

Figure 4: Evolution of $p a^{2} / \pi^{2} D$ with respect to $Z=\sqrt{1-v^{2}} a^{2} / R h$ for panels with: (a) $b / a=0.5$; (b) $b / a=1$; (c) $b / a=2$.

Author's Contribuitions: Conceptualization, E Lucena Neto; Methodology, E Lucena Neto and FAC Monteiro; Investigation, PTM Soares, RC Santana, E Lucena Neto, FAC Monteiro, AF Souza Neto and PIB Queiroz; Software: PTM Soares, RC Santana, FAC Monteiro; Writing - original draft, E Lucena Neto and FAC Monteiro; Writing - review \& editing, E Lucena Neto and FAC Monteiro; Supervision, E Lucena Neto.

Guest Editors: Volnei Tita and Nicholas Fantuzzi. 


\section{References}

Afkhami, S.E., Vahid, M., Niazi, M., Abbasi, M. (2015). Analytical and numerical investigations on buckling of an axially compressed cylindrical panel with specific boundary condition, International Review of Mechanical Engineering 9(2):118-123.

Bardell, N.S. (1991). Free vibration analysis of a flat plate using the hierarchical finite element method, Journal of Sound Vibration 151 (2): 263-289.

Brush, D.O., Almroth, B.O. (1975). Buckling of Bars, Plates, and Shells, McGraw-Hill (New York).

Buermann, P., Rolfes, R., Tessmer, J., Schagerl, M. (2006). A semi-analytical model for local post-buckling analysis of stringerand frame-stiffened cylindrical panels, Thin-Walled Structures 44(1):102-114.

Chu, K.H., Krishnamoorthy, G. (1967). Buckling of open cylindrical shells, Journal of the Engineering Mechanics Division, 93(EM 2):177-205.

Eipakchi, H.R., Shariati, M. (2011). Buckling analysis of a cylindrical panel under axial stress using perturbation technique, Journal of Applied Mathematics and Mechanics 91(2):138-145.

Gelfand, I.M., Fomin, S.V. (2000). Calculus of Variations, Dover (Mineola).

Kang, K., Bert, C.W., Striz, A.G. (1996). Vibration analysis of horizontally curved beams with warping using DQM, Journal of Structural Engineering 122(6):657-662.

King, B.R. (1996). Beyond the Quartic Equation, Birkhäuser (Boston).

Koiter, W.T. (1956). Buckling and post-buckling behaviour of a cylindrical panel under axial compression, Rep. No. NLL-TR S. 476, National Aerospace Research Institute (Amsterdam).

Kollár, L., Dulácska, E. (1984). Buckling of Shells for Engineers, Wiley (Chichester).

Kollbrunner, C.F., Basler, K. (1969). Torsion in Structures, Springer (Berlin).

Krishnamoorthy, G. (1974). Buckling of open cylindrical shells with torsionally stiff rectangular edge Stiffeners, AIAA Journal 12(10):1348-1353.

Lucena Neto, E., Monteiro, F.A.C., Soares, P.T.M.L. (2016). Exact solution for buckling of axially compressed cylindrical panels with stringers attached to the straight edges, Journal of Engineering Mechanics 142(16):04016028.

Magnucki, K., Maćkiewicz, M. (2006). Elastic buckling of an axially compressed cylindrical panel with three edges simply supported and one edge free, Thin-Walled Structures 44(4):387-392.

Martins, J.P., Simões da Silva, L., Reis, A. (2013). Eigenvalue analysis of cylindrically curved panels under compressive stresses Extension of rules from EN 1993-1-5, Thin-Walled Structures 68:183-194.

Martins, J.P., Ljubinkovic, F., Simões da Silva, L., Gervásio, H. (2018). Behaviour of thin-walled curved steel plates under generalised in-plane stresses: a review, Journal of Constructional Steel Research 140:191-207.

MATLAB (2016), version R2016a, MathWorks (Natick).

McNamee, J.M. (2007). Numerical Methods for Roots of Polynomials, Part I, Elsevier (Amsterdam).

McNamee, J.M., Pan, V.Y. (2013). Numerical Methods for Roots of Polynomials, Part II, Elsevier (Amsterdam).

Peterson, J.P., Seide, P., Weingarten, V.I. (1968). Buckling of thin-walled circular cylinders, Report NASA-SP-8007, National Aeronautics and Space Administration (Hampton).

Pilkey, W.D. (2002). Analysis and Design of Elastic Beams: Computational Methods, John Wiley (New York).

Sosa, E.M., Godoy, L.A., Croll, J.G.A. (2006). Computation of lower-bound elastic buckling loads using general-purpose finite element codes, Computers and Structures 84(29-30):1934-1945.

Stephens, W.B. (1971). Imperfection sensitivity of axially compressed stringer reinforced cylindrical panels under internal pressure, AIAA J. 9(9):1713-1719.

Singer, J. (2004). Stiffened cylindrical shells, In: Teng, J.G., Rotter, J.M., eds., Buckling of Thin Metal Shells, Spon Press (London), 286-343. 
Timoshenko, S.P., Gere, J.M. (1961). Theory of Elastic Stability, 2nd edn., McGraw-Hill (New York).

Tran, K.L, Davaine, L., Douthe, C., Sab, K. (2012). Stability of curved panels under uniform axial compression, Journal of Constructional Steel Research 69:30-38.

Wilde, R., Zawodny, P., Magnucki, K. (2007). Critical state of an axially compressed cylindrical panel with three edges simply supported and one edge free, Thin-Walled Structures 45(10-11):955-959.

Yshii, L.N., Lucena Neto, N., Monteiro, F.A.C., Santana, R.C. (2018). Accuracy of the buckling predictions of anisotropic plates, Journal of Engineering Mechanics 144(8):04018061. 


\section{APPENDIX A. EXPRESSION OF THE EXTERNAL MOMENT $\bar{M}$}

Figure 5 shows an infinitesimal portion of the frame attached to the panel edge $x=-a / 2$, under the action of the external moment $\bar{M}(-a / 2, y)$ exerted by the panel and the internal force and moments due to the torsion of the frame. One finds on summing forces in the out-of-plane direction

$$
-Q+Q+d Q=0 \quad \Rightarrow \quad d Q=0 \quad \Rightarrow \quad Q_{, y}=0 .
$$

The sum of moments about a vertical axis gives

$$
-M_{t} \sin \frac{d \theta}{2}-\left(M_{t}+d M_{t}\right) \sin \frac{d \theta}{2}-M \cos \frac{d \theta}{2}+(M+d M) \cos \frac{d \theta}{2}-Q d y=0
$$

or

$$
M_{, y}-\frac{M_{t}}{R}-Q=0
$$

by using $\cos d \theta / 2 \approx 1$ and $\sin d \theta / 2 \approx d \theta / 2$ and neglecting higher order terms. Similarly, summing moments about a horizontal axis yields

$$
\frac{M}{R}+M_{t, y}+\bar{M}=0
$$

Note that the torsion and out-of-plane bending are coupled in the equilibrium equations (A.1), (A.3) and (A.4).

Based on the fundamental lemma of variational calculus (Gelfand and Fomin, 2000), the principle of virtual displacement associated with this problem must have the form

$$
\int_{0}^{b}\left[Q_{, y} \delta u+\left(M_{, y}-\frac{M_{t}}{R}-Q\right) \delta \theta_{z}+\left(\frac{M}{R}+M_{t, y}+\bar{M}\right) \delta \theta_{y}\right] d y+\cdots=0
$$

The boundary terms are not shown, $\theta_{y}$ and $\theta_{z}$ are the rotations about the $y$ and $z$ axes, and the coefficients of $\delta u, \delta \theta_{z}$ and $\delta \theta_{y}$ are the equilibrium equations (A.1), (A.3) and (A.4), respectively. It is convenient to write (Pilkey, 2002)

$$
M_{t}=M_{s v}-M_{\omega, y}
$$

in which $M_{s v}$ and $-M_{\omega, y}$ are the Saint-Venant and warping torsions ( $M_{\omega}$ denotes the bimoment). Thus,

$$
\int_{0}^{b}\left[Q, y \delta u+\left(M_{, y}-\frac{M_{s v}}{R}+\frac{M_{\omega, y}}{R}-Q\right) \delta \theta_{z}+\left(\frac{M}{R}+M_{s v, y}-M_{\omega, y y}+\bar{M}\right) \delta \theta_{y}\right] d y+\cdots=0
$$

or, using integration by parts,

$$
-\int_{0}^{b}\left[M \delta\left(-\frac{\theta_{y}}{R}+\theta_{z, y}\right)+Q \delta\left(u_{, y}+\theta_{z}\right)+M_{s v} \delta\left(\theta_{y, y}+\frac{\theta_{z}}{R}\right)+M_{\omega} \delta\left(\theta_{y, y y}+\frac{\theta_{z, y}}{R}\right)-\bar{M} \delta \theta_{y}\right] d y+\cdots=0 .
$$

One can identify

$$
-\int_{0}^{b}\left[M \delta\left(-\frac{\theta_{y}}{R}+\theta_{z, y}\right)+Q \delta\left(u_{, y}+\theta_{z}\right)+M_{s v} \delta\left(\theta_{y, y}+\frac{\theta_{z}}{R}\right)+M_{\omega} \delta\left(\theta_{y, y y}+\frac{\theta_{z, y}}{R}\right)\right] d y
$$

as the internal virtual work, which suggests that 


$$
-\frac{\theta_{y}}{R}+\theta_{z, y} \quad u_{, y}+\theta_{z} \quad \theta_{y, y}+\frac{\theta_{z}}{R} \quad \theta_{y, y y}+\frac{\theta_{z, y}}{R}
$$

are the work-conjugate strains to $M, Q, M_{s v}$ and $M_{\omega}$, respectively.

The second strain in (A.9) can be eliminated by the Euler-Bernoulli assumption $\theta_{z}=-u, y$. The constitutive equations are then given by

$$
M=-E_{f} I_{f}\left(u_{, y y}+\frac{\theta_{y}}{R}\right) \quad M_{s v}=G_{f} J_{f}\left(\theta_{y, y}-\frac{u_{, y}}{R}\right) \quad M_{\omega}=E_{f} \Gamma_{f}\left(\theta_{y, y y}-\frac{u_{, y y}}{R}\right) .
$$

Substitution of (A.6) and (A.11) into (A.4), replacing the frame twist $\theta_{y}$ by $-w_{, x}$, gives finally

$$
\bar{M}=\frac{E_{f} I_{f}}{R}\left(u_{, y y}-\frac{w_{, x}}{R}\right)+G_{f} J_{f}\left(\frac{u, y y}{R}+w_{, x y y}\right)-E_{f} \Gamma_{f}\left(\frac{u, y y y y}{R}+w_{, x y y y y}\right) .
$$

A similar expression can be found in (Kang et al., 1996) in the context of vibrations of curved beams. The sense of $\bar{M}$ shown in Figure 5 must be reversed for the frame attached to the panel edge $x=a / 2$.

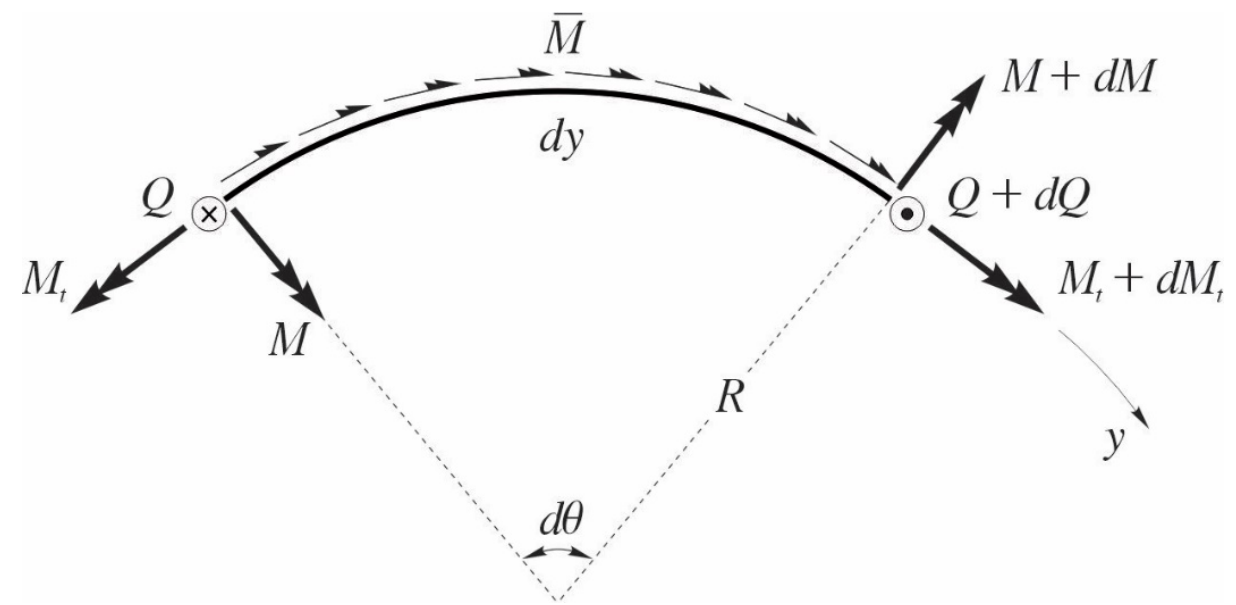

Figure 5: Infinitesimal portion of the frame attached to the panel edge $x=-a / 2$.

\section{APPENDIX B. DISPLACEMENT COEFFICIENTS AND MATRIX K}

The coefficients $u_{i s}, \bar{u}_{i s}, u_{i a}, \bar{u}_{i a}, v_{i s}, \bar{v}_{i s}, v_{i a}, \bar{v}_{i a}$ and entries of matrix $\mathbf{K}$ are listed in the sequel.

\section{B.1 Case I}

Symmetric modes:

$$
\begin{aligned}
& u_{i s}=\gamma_{i} \frac{k^{2}-v \gamma_{i}^{2}}{\left(k^{2}+\gamma_{i}^{2}\right)^{2}} \quad v_{i s}=k \frac{k^{2}+(2+v) \gamma_{i}^{2}}{\left(k^{2}+\gamma_{i}^{2}\right)^{2}} \quad i=1,2,3,4 \\
& k_{1 i}=u_{i s} \gamma_{i} \cos \gamma_{i} \xi_{0} \quad k_{2 i}=v_{i s} \cos \gamma_{i} \xi_{0} \quad k_{3 i}=\cos \gamma_{i} \xi_{0} \\
& k_{4 i}=-\frac{D}{R} \gamma_{i}^{2} \cos \gamma_{i} \xi_{0}-A \gamma_{i} \sin \gamma_{i} \xi_{0}+B u_{i s} \sin \gamma_{i} \xi_{0} \quad i=1,2,3,4
\end{aligned}
$$

in which

$$
A=\sqrt{\frac{p_{c l}}{2 E h}}\left(\frac{E_{f} I_{f}}{R^{2}}+\frac{n^{2} \pi^{2}}{b^{2}} G_{f} J_{f}+\frac{n^{4} \pi^{4}}{b^{4}} E_{f} \Gamma_{f}\right) \quad B=\frac{n^{2} \pi^{2}}{b^{2}}\left(\frac{p_{c l}}{2 E h}\right)^{3 / 2}\left(E_{f} I_{f}+G_{f} J_{f}+\frac{n^{2} \pi^{2}}{b^{2}} E_{f} \Gamma_{f}\right) .
$$


Antisymmetric modes:

$$
\begin{aligned}
& \left(u_{i a}, v_{i a}\right)=\left(-u_{i s}, v_{i s}\right) \quad i=1,2,3,4 \\
& k_{1 i}=-u_{i a} \gamma_{i} \sin \gamma_{i} \xi_{0} \quad k_{2 i}=v_{i a} \sin \gamma_{i} \xi_{0} \quad k_{3 i}=\sin \gamma_{i} \xi_{0} \\
& k_{4 i}=-\frac{D}{R} \gamma_{i}^{2} \sin \gamma_{i} \xi_{0}+A \gamma_{i} \cos \gamma_{i} \xi_{0}+B u_{i a} \cos \gamma_{i} \xi_{0} \quad i=1,2,3,4
\end{aligned}
$$

\section{B.2 Case II}

Symmetric modes:

$$
\begin{aligned}
& \left(u_{1 s}, u_{3 s}, u_{4 s}, v_{1 s}, v_{3 s}, v_{4 s}\right)=\left(u_{1 s}, u_{3 s}, u_{4 s}, v_{1 s}, v_{3 s}, v_{4 s}\right) \text { of Case I } \\
& u_{2 s}=\frac{1+v}{4 k^{2}} \quad v_{2 s}=\frac{1}{2 k^{2}} \quad\left(\bar{u}_{2 s}, \bar{v}_{2 s}\right)=\left(-u_{1 s}, v_{1 s}\right) \\
& k_{1 i}=u_{i s} \gamma_{i} \cos \gamma_{i} \xi_{0} \quad k_{12}=u_{2 s} \gamma_{1} \cos \gamma_{1} \xi_{0}+\bar{u}_{2 s}\left(\cos \gamma_{1} \xi_{0}-\gamma_{1} \xi_{0} \sin \gamma_{1} \xi_{0}\right) \\
& k_{2 i}=v_{i s} \cos \gamma_{i} \xi_{0} \quad k_{32}=v_{2 s} \cos \gamma_{1} \xi_{0}+\bar{v}_{2 s} \xi_{0} \sin \gamma_{1} \xi_{0} \\
& k_{3 i}=\cos \gamma_{i} \xi_{0} \quad \\
& k_{4 i}=-\frac{D}{R} \gamma_{i}^{2} \cos \gamma_{i} \xi_{0}-A \gamma_{i} \sin \gamma_{i} \xi_{0}+B u_{i s} \sin \gamma_{i} \xi_{0} \\
& k_{42}=\frac{D}{R} \gamma_{1}\left(2 \cos \gamma_{1} \xi_{0}-\gamma_{1} \xi_{0} \sin \gamma_{1} \xi_{0}\right)+A\left(\sin \gamma_{1} \xi_{0}+\gamma_{1} \xi_{0} \cos \gamma_{1} \xi_{0}\right) \\
& +B\left(u_{2 s} \sin \gamma_{1} \xi_{0}+\bar{u}_{2 s} \xi_{0} \cos \gamma_{1} \xi_{0}\right) \quad i=1,3,4 .
\end{aligned}
$$

Antisymmetric modes:

$$
\begin{aligned}
& \left(u_{1 a}, u_{3 a}, u_{4 a}, v_{1 a}, v_{3 a}, v_{4 a}\right)=\left(u_{1 a}, u_{3 a}, u_{4 a}, v_{1 a}, v_{3 a}, v_{4 a}\right) \text { of Case I } \\
& \quad\left(u_{2 a}, \bar{u}_{2 a}, v_{2 a}, \bar{v}_{2 a}\right)=\left(u_{2 s},-\bar{u}_{2 s},-v_{2 s}, \bar{v}_{2 s}\right) \\
& k_{1 i}=-u_{i a} \gamma_{i} \sin \gamma_{i} \xi_{0} \quad k_{12}=-u_{2 a} \gamma_{1} \sin \gamma_{1} \xi_{0}+\bar{u}_{2 a}\left(\sin \gamma_{1} \xi_{0}+\gamma_{1} \xi_{0} \cos \gamma_{1} \xi_{0}\right) \\
& k_{2 i}=v_{i a} \sin \gamma_{i} \xi_{0} \quad k_{22}=v_{2 a} \sin \gamma_{1} \xi_{0}+\bar{v}_{2 a} \xi_{0} \cos \gamma_{1} \xi_{0} \\
& k_{3 i}=\sin \gamma_{i} \xi_{0} \quad \xi_{0} \cos \gamma_{1} \xi_{0} \\
& k_{4 i}=-\frac{D}{R} \gamma_{i}^{2} \sin \gamma_{i} \xi_{0}+A \gamma_{i} \cos \gamma_{i} \xi_{0}+B u_{i a} \cos \gamma_{i} \xi_{0} \\
& k_{42}=-\frac{D}{R} \gamma_{1}\left(2 \sin \gamma_{1} \xi_{0}+\gamma_{1} \xi_{0} \cos \gamma_{1} \xi_{0}\right)+A\left(\cos \gamma_{1} \xi_{0}-\gamma_{1} \xi_{0} \sin \gamma_{1} \xi_{0}\right) \\
& +B\left(u_{2 a} \cos \gamma_{1} \xi_{0}+\bar{u}_{2 a} \xi_{0} \sin \gamma_{1} \xi_{0}\right) \quad i=1,3,4 .
\end{aligned}
$$

\section{B.3 Case III}

Symmetric modes: 
$\left(u_{3 s}, u_{4 s}, v_{3 s}, v_{4 s}\right)=\left(u_{3 s}, u_{4 s}, v_{3 s}, v_{4 s}\right)$ of Case I

$$
\begin{aligned}
& u_{1 s}=\frac{v-1}{4 \beta_{1}} \quad \bar{u}_{1 s}=\frac{(1+v) \alpha_{1}}{4 \beta_{1}^{2}} \quad\left(u_{2 s}, \bar{u}_{2 s}\right)=\left(\bar{u}_{1 s},-u_{1 s}\right) \\
& v_{1 s}=\frac{1}{2 k}+\frac{(1+v) k}{4 \beta_{1}^{2}} \quad \bar{v}_{1 s}=\frac{\alpha_{1}}{2 k \beta_{1}} \quad\left(v_{2 s}, \bar{v}_{2 s}\right)=\left(-\bar{v}_{1 s}, v_{1 s}\right)
\end{aligned}
$$

$k_{1 i}=\left(\alpha_{1} u_{i s}+\beta_{1} \bar{u}_{i s}\right) \cosh \alpha_{1} \xi_{0} \cos \beta_{1} \xi_{0}+\left(\alpha_{1} \bar{u}_{i s}-\beta_{1} u_{i s}\right) \sinh \alpha_{1} \xi_{0} \sin \beta_{1} \xi_{0} \quad i=1,2$

$k_{1 i}=u_{i s} \gamma_{i} \cos \gamma_{i} \xi_{0} \quad i=3,4$

$k_{2 i}=v_{i s} \sinh \alpha_{1} \xi_{0} \sin \beta_{1} \xi_{0}+\bar{v}_{i s} \cosh \alpha_{1} \xi_{0} \cos \beta_{1} \xi_{0} \quad i=1,2$

$k_{2 i}=v_{i s} \cos \gamma_{i} \xi_{0} \quad i=3,4$

$k_{31}=\sinh \alpha_{1} \xi_{0} \sin \beta_{1} \xi_{0} \quad k_{32}=\cosh \alpha_{1} \xi_{0} \cos \beta_{1} \xi_{0} \quad k_{3 i}=\cos \gamma_{i} \xi_{0} \quad i=3,4$

$k_{41}=\frac{D}{R}\left[\left(\alpha_{1}^{2}-\beta_{1}^{2}\right) \sinh \alpha_{1} \xi_{0} \sin \beta_{1} \xi_{0}+2 \alpha_{1} \beta_{1} \cosh \alpha_{1} \xi_{0} \cos \beta_{1} \xi_{0}\right]$

$+\left(A \alpha_{1}+B \bar{u}_{1 s}\right) \cosh \alpha_{1} \xi_{0} \sin \beta_{1} \xi_{0}+\left(A \beta_{1}+B u_{1 s}\right) \sinh \alpha_{1} \xi_{0} \cos \beta_{1} \xi_{0}$

$k_{42}=\frac{D}{R}\left[\left(\alpha_{1}^{2}-\beta_{1}^{2}\right) \cosh \alpha_{1} \xi_{0} \cos \beta_{1} \xi_{0}-2 \alpha_{1} \beta_{1} \sinh \alpha_{1} \xi_{0} \sin \beta_{1} \xi_{0}\right]$

$+\left(A \alpha_{1}+B u_{2 s}\right) \sinh \alpha_{1} \xi_{0} \cos \beta_{1} \xi_{0}-\left(A \beta_{1}-B \bar{u}_{2 s}\right) \cosh \alpha_{1} \xi_{0} \sin \beta_{1} \xi_{0}$

$k_{4 i}=-\frac{D}{R} \gamma_{i}^{2} \cos \gamma_{i} \xi_{0}-A \gamma_{i} \sin \gamma_{i} \xi_{0}+B u_{i s} \sin \gamma_{i} \xi_{0} \quad i=3,4$

\section{Antisymmetric modes:}

$$
\begin{gathered}
\left(u_{1 a}, \bar{u}_{1 a}, u_{2 a}, \bar{u}_{2 a}, v_{1 a}, \bar{v}_{1 a}, v_{2 a}, \bar{v}_{2 a}\right)=\left(-u_{1 s}, \bar{u}_{1 s}, \bar{u}_{1 s}, u_{1 s}, v_{1 s},-\bar{v}_{1 s}, \bar{v}_{1 s}, v_{1 s}\right) \\
\left(u_{3 a}, u_{4 a}, v_{3 a}, v_{4 a}\right)=\left(u_{3 a}, u_{4 a}, v_{3 a}, v_{4 a}\right) \text { of Case I }
\end{gathered}
$$

$k_{1 i}=\left(\alpha_{1} u_{i a}-\beta_{1} \bar{u}_{i a}\right) \cosh \alpha_{1} \xi_{0} \sin \beta_{1} \xi_{0}+\left(\alpha_{1} \bar{u}_{i a}+\beta_{1} u_{i a}\right) \sinh \alpha_{1} \xi_{0} \cos \beta_{1} \xi_{0} \quad i=1,2$

$k_{1 i}=-u_{i a} \gamma_{i} \sin \gamma_{i} \xi_{0} \quad i=3,4$

$k_{2 i}=v_{i a} \sinh \alpha_{1} \xi_{0} \cos \beta_{1} \xi_{0}+\bar{v}_{i a} \cosh \alpha_{1} \xi_{0} \sin \beta_{1} \xi_{0} \quad i=1,2$

$k_{2 i}=v_{i a} \sin \gamma_{i} \xi_{0} \quad i=3,4$

$k_{31}=\sinh \alpha_{1} \xi_{0} \cos \beta_{1} \xi_{0} \quad k_{32}=\cosh \alpha_{1} \xi_{0} \sin \beta_{1} \xi_{0} \quad k_{3 i}=\sin \gamma_{i} \xi_{0} \quad i=3,4$

$k_{41}=\frac{D}{R}\left[\left(\alpha_{1}^{2}-\beta_{1}^{2}\right) \sinh \alpha_{1} \xi_{0} \cos \beta_{1} \xi_{0}-2 \alpha_{1} \beta_{1} \cosh \alpha_{1} \xi_{0} \sin \beta_{1} \xi_{0}\right]$

$+\left(A \alpha_{1}+B \bar{u}_{1 a}\right) \cosh \alpha_{1} \xi_{0} \cos \beta_{1} \xi_{0}-\left(A \beta_{1}-B u_{1 a}\right) \sinh \alpha_{1} \xi_{0} \sin \beta_{1} \xi_{0}$ 


$$
\begin{aligned}
k_{42}= & \frac{D}{R}\left[\left(\alpha_{1}^{2}-\beta_{1}^{2}\right) \cosh \alpha_{1} \xi_{0} \sin \beta_{1} \xi_{0}+2 \alpha_{1} \beta_{1} \sinh \alpha_{1} \xi_{0} \cos \beta_{1} \xi_{0}\right] \\
& +\left(A \alpha_{1}+B u_{2 a}\right) \sinh \alpha_{1} \xi_{0} \sin \beta_{1} \xi_{0}+\left(A \beta_{1}+B \bar{u}_{2 a}\right) \cosh \alpha_{1} \xi_{0} \cos \beta_{1} \xi_{0} \\
k_{4 i}= & -\frac{D}{R} \gamma_{i}^{2} \sin \gamma_{i} \xi_{0}+A \gamma_{i} \cos \gamma_{i} \xi_{0}+B u_{i a} \cos \gamma_{i} \xi_{0} \quad i=3,4 .
\end{aligned}
$$

\section{B.4 Case IV}

Symmetric modes:

$$
\begin{aligned}
\left(u_{1 s}, \bar{u}_{1 s}, u_{2 s}, \bar{u}_{2 s}, v_{1 s}, \bar{v}_{1 s}, v_{2 s}, \bar{v}_{2 s}\right) & =\left(u_{1 s}, \bar{u}_{1 s}, u_{2 s}, \bar{u}_{2 s}, v_{1 s}, \bar{v}_{1 s}, v_{2 s}, \bar{v}_{2 s}\right) \text { of Case III } \\
\left(u_{3 s}, v_{3 s}\right) & =\left(u_{3 s}, v_{3 s}\right) \text { of Case I. }
\end{aligned}
$$

The coefficients $\left(u_{4 s}, \bar{u}_{4 s}, v_{4 s}, \bar{v}_{4 s}\right)$ remain the same as those of $\left(u_{2 s}, \bar{u}_{2 s}, v_{2 s}, \bar{v}_{2 s}\right)$ found in Case II with $\gamma_{1}$ replaced by $\gamma_{3}$.

$$
\begin{aligned}
k_{1 i}= & \left(\alpha_{1} u_{i s}+\beta_{1} \bar{u}_{i s}\right) \cosh \alpha_{1} \xi_{0} \cos \beta_{1} \xi_{0}+\left(\alpha_{1} \bar{u}_{i s}-\beta_{1} u_{i s}\right) \sinh \alpha_{1} \xi_{0} \sin \beta_{1} \xi_{0} \quad k_{14}=u_{4 s} \gamma_{3} \cos \gamma_{3} \xi_{0}+\bar{u}_{4 s}\left(\cos \gamma_{3} \xi_{0}-\gamma_{3} \xi_{0} \sin \gamma_{3} \xi_{0}\right) \\
k_{13}= & u_{3 s} \gamma_{3} \cos \gamma_{3} \xi_{0} \quad k_{24}=v_{4 s} \cos \gamma_{3} \xi_{0}+\bar{v}_{4 s} \xi_{0} \sin \gamma_{3} \xi_{0} \\
k_{2 i}= & v_{i s} \sinh \alpha_{1} \xi_{0} \sin \beta_{1} \xi_{0}+\bar{v}_{i s} \cosh \alpha_{1} \xi_{0} \cos \beta_{1} \xi_{0} \quad i=1,2 \\
k_{23}= & v_{3 s} \cos \gamma_{3} \xi_{0} \quad k_{32}=\cosh \alpha_{1} \xi_{0} \cos \beta_{1} \xi_{0} \\
k_{31}= & \sinh \alpha_{1} \xi_{0} \sin \beta_{1} \xi_{0} \quad \sin \gamma_{3} \xi_{0} \\
k_{33}= & \cos \gamma_{3} \xi_{0} \quad \\
k_{41}= & \frac{D}{R}\left[\left(\alpha_{1}^{2}-\beta_{1}^{2}\right) \sinh \alpha_{1} \xi_{0} \sin \beta_{1} \xi_{0}+2 \alpha_{1} \beta_{1} \cosh \alpha_{1} \xi_{0} \cos \beta_{1} \xi_{0}\right] \\
& +\left(A \alpha_{1}+B \bar{u}_{1 s}\right) \cosh \alpha_{1} \xi_{0} \sin \beta_{1} \xi_{0}+\left(A \beta_{1}+B u_{1 s}\right) \sinh \alpha_{1} \xi_{0} \cos \beta_{1} \xi_{0} \\
k_{42}= & \frac{D}{R}\left[\left(\alpha_{1}^{2}-\beta_{1}^{2}\right) \cosh \alpha_{1} \xi_{0} \cos \beta_{1} \xi_{0}-2 \alpha_{1} \beta_{1} \sinh \alpha_{1} \xi_{0} \sin \beta_{1} \xi_{0}\right] \\
& +\left(A \alpha_{1}+B u_{2 s}\right) \sinh \alpha_{1} \xi_{0} \cos \beta_{1} \xi_{0}-\left(A \beta_{1}-B \bar{u}_{2 s}\right) \cosh \alpha_{1} \xi_{0} \sin \beta_{1} \xi_{0} \\
& \\
k_{43}= & -\frac{D}{R} \gamma_{3}^{2} \cos \gamma_{3} \xi_{0}-A \gamma_{3} \sin \gamma_{3} \xi_{0}+B u_{3 s} \sin \gamma_{3} \xi_{0} \\
k_{44}= & \frac{D}{R} \gamma_{3}\left(2 \cos \gamma_{3} \xi_{0}-\gamma_{3} \xi_{0} \sin \gamma_{3} \xi_{0}\right)+A\left(\sin \gamma_{3} \xi_{0}+\gamma_{3} \xi_{0} \cos \gamma_{3} \xi_{0}\right)+B\left(u_{4 s} \sin \gamma_{3} \xi_{0}+\bar{u}_{4 s} \xi_{0} \cos \gamma_{3} \xi_{0}\right)
\end{aligned}
$$

Antisymmetric modes:

$$
\begin{aligned}
\left(u_{1 a}, \bar{u}_{1 a}, u_{2 a}, \bar{u}_{2 a}, v_{1 a}, \bar{v}_{1 a}, v_{2 a}, \bar{v}_{2 a}\right) & =\left(u_{1 a}, \bar{u}_{1 a}, u_{2 a}, \bar{u}_{2 a}, v_{1 a}, \bar{v}_{1 a}, v_{2 a}, \bar{v}_{2 a}\right) \text { of Case III } \\
\left(u_{3 a}, v_{3 a}\right) & =\left(u_{3 a}, v_{3 a}\right) \text { of Case I. }
\end{aligned}
$$

The coefficients $\left(u_{4 a}, \bar{u}_{4 a}, v_{4 a}, \bar{v}_{4 a}\right)$ remain the same as those of $\left(u_{2 a}, \bar{u}_{2 a}, v_{2 a}, \bar{v}_{2 a}\right)$ found in Case II with $\gamma_{1}$ replaced by $\gamma_{3}$.

$$
k_{1 i}=\left(\alpha_{1} u_{i a}-\beta_{1} \bar{u}_{i a}\right) \cosh \alpha_{1} \xi_{0} \sin \beta_{1} \xi_{0}+\left(\alpha_{1} \bar{u}_{i a}+\beta_{1} u_{i a}\right) \sinh \alpha_{1} \xi_{0} \cos \beta_{1} \xi_{0} \quad i=1,2
$$




$$
\begin{aligned}
& k_{13}=-u_{3 a} \gamma_{3} \sin \gamma_{3} \xi_{0} \quad k_{14}=-u_{4 a} \gamma_{3} \sin \gamma_{3} \xi_{0}+\bar{u}_{4 a}\left(\sin \gamma_{3} \xi_{0}+\gamma_{3} \xi_{0} \cos \gamma_{3} \xi_{0}\right) \\
& k_{2 i}=v_{i a} \sinh \alpha_{1} \xi_{0} \cos \beta_{1} \xi_{0}+\bar{v}_{i a} \cosh \alpha_{1} \xi_{0} \sin \beta_{1} \xi_{0} \quad i=1,2 \\
& k_{23}=v_{3 a} \sin \gamma_{3} \xi_{0} \quad k_{24}=v_{4 a} \sin \gamma_{3} \xi_{0}+\bar{v}_{4 a} \xi_{0} \cos \gamma_{3} \xi_{0} \\
& k_{31}=\sinh \alpha_{1} \xi_{0} \cos \beta_{1} \xi_{0} \quad k_{32}=\cosh \alpha_{1} \xi_{0} \sin \beta_{1} \xi_{0} \\
& k_{33}=\sin \gamma_{3} \xi_{0} \quad k_{34}=\xi_{0} \cos \gamma_{3} \xi_{0} \\
& k_{41}=\frac{D}{R}\left[\left(\alpha_{1}^{2}-\beta_{1}^{2}\right) \sinh \alpha_{1} \xi_{0} \cos \beta_{1} \xi_{0}-2 \alpha_{1} \beta_{1} \cosh \alpha_{1} \xi_{0} \sin \beta_{1} \xi_{0}\right] \\
& +\left(A \alpha_{1}+B \bar{u}_{1 a}\right) \cosh \alpha_{1} \xi_{0} \cos \beta_{1} \xi_{0}-\left(A \beta_{1}-B u_{1 a}\right) \sinh \alpha_{1} \xi_{0} \sin \beta_{1} \xi_{0} \\
& k_{42}=\frac{D}{R}\left[\left(\alpha_{1}^{2}-\beta_{1}^{2}\right) \cosh \alpha_{1} \xi_{0} \sin \beta_{1} \xi_{0}+2 \alpha_{1} \beta_{1} \sinh \alpha_{1} \xi_{0} \cos \beta_{1} \xi_{0}\right] \\
& +\left(A \alpha_{1}+B u_{2 a}\right) \sinh \alpha_{1} \xi_{0} \sin \beta_{1} \xi_{0}+\left(A \beta_{1}+B \bar{u}_{2 a}\right) \cosh \alpha_{1} \xi_{0} \cos \beta_{1} \xi_{0} \\
& k_{43}=-\frac{D}{R} \gamma_{3}^{2} \sin \gamma_{3} \xi_{0}+A \gamma_{3} \cos \gamma_{3} \xi_{0}+B u_{3 a} \cos \gamma_{3} \xi_{0} \\
& k_{44}=-\frac{D}{R} \gamma_{3}\left(2 \sin \gamma_{3} \xi_{0}+\gamma_{3} \xi_{0} \cos \gamma_{3} \xi_{0}\right)+A\left(\cos \gamma_{3} \xi_{0}-\gamma_{3} \xi_{0} \sin \gamma_{3} \xi_{0}\right) \\
& +B\left(u_{4 a} \cos \gamma_{3} \xi_{0}+\bar{u}_{4 a} \xi_{0} \sin \gamma_{3} \xi_{0}\right) \text {. }
\end{aligned}
$$

\section{B.5 Case V}

Symmetric modes:

$$
\left(u_{1 s}, \bar{u}_{1 s}, u_{2 s}, \bar{u}_{2 s}, v_{1 s}, \bar{v}_{1 s}, v_{2 s}, \bar{v}_{2 s}\right)=\left(u_{1 s}, \bar{u}_{1 s}, u_{2 s}, \bar{u}_{2 s}, v_{1 s}, \bar{v}_{1 s}, v_{2 s}, \bar{v}_{2 s}\right) \text { of Case III. }
$$

The coefficients $\left(u_{3 s}, \bar{u}_{3 s}, v_{3 s}, \bar{v}_{3 s}\right)$ and $\left(u_{4 s}, \bar{u}_{4 s}, v_{4 s}, \bar{v}_{4 s}\right)$ remain the same as those of $\left(u_{1 s}, \bar{u}_{1 s}, v_{1 s}, \bar{v}_{1 s}\right)$ and $\left(u_{2 s}, \bar{u}_{2 s}\right.$, $\left.v_{2 s}, \bar{v}_{2 s}\right)$, respectively, with $\alpha_{1}$ and $\beta_{1}$ replaced by $\alpha_{2}$ and $\beta_{2}$.

$$
\begin{aligned}
k_{1 i}= & \left(\alpha_{1} u_{i s}+\beta_{1} \bar{u}_{i s}\right) \cosh \alpha_{1} \xi_{0} \cos \beta_{1} \xi_{0}+\left(\alpha_{1} \bar{u}_{i s}-\beta_{1} u_{i s}\right) \sinh \alpha_{1} \xi_{0} \sin \beta_{1} \xi_{0} \quad i=1,2 \\
k_{1 i}= & \left(\alpha_{2} u_{i s}+\beta_{2} \bar{u}_{i s}\right) \cosh \alpha_{2} \xi_{0} \cos \beta_{2} \xi_{0}+\left(\alpha_{2} \bar{u}_{i s}-\beta_{2} u_{i s}\right) \sinh \alpha_{2} \xi_{0} \sin \beta_{2} \xi_{0} \quad i=3,4 \\
k_{2 i}= & v_{i s} \sinh \alpha_{1} \xi_{0} \sin \beta_{1} \xi_{0}+\bar{v}_{i s} \cosh \alpha_{1} \xi_{0} \cos \beta_{1} \xi_{0} \quad i=1,2 \\
k_{2 i}= & v_{i s} \sinh \alpha_{2} \xi_{0} \sin \beta_{2} \xi_{0}+\bar{v}_{i s} \cosh \alpha_{2} \xi_{0} \cos \beta_{2} \xi_{0} \quad i=3,4 \\
k_{31}= & \sinh \alpha_{1} \xi_{0} \sin \beta_{1} \xi_{0} \quad k_{32}=\cosh \alpha_{1} \xi_{0} \cos \beta_{1} \xi_{0} \\
k_{33}= & \sinh \alpha_{2} \xi_{0} \sin \beta_{2} \xi_{0} \quad k_{34}=\cosh \alpha_{2} \xi_{0} \cos \beta_{2} \xi_{0} \\
k_{41}= & \frac{D}{R}\left[\left(\alpha_{1}^{2}-\beta_{1}^{2}\right) \sinh \alpha_{1} \xi_{0} \sin \beta_{1} \xi_{0}+2 \alpha_{1} \beta_{1} \cosh \alpha_{1} \xi_{0} \cos \beta_{1} \xi_{0}\right] \\
& +\left(A \alpha_{1}+B \bar{u}_{1 s}\right) \cosh \alpha_{1} \xi_{0} \sin \beta_{1} \xi_{0}+\left(A \beta_{1}+B u_{1 s}\right) \sinh \alpha_{1} \xi_{0} \cos \beta_{1} \xi_{0}
\end{aligned}
$$




$$
\begin{aligned}
k_{42}= & \frac{D}{R}\left[\left(\alpha_{1}^{2}-\beta_{1}^{2}\right) \cosh \alpha_{1} \xi_{0} \cos \beta_{1} \xi_{0}-2 \alpha_{1} \beta_{1} \sinh \alpha_{1} \xi_{0} \sin \beta_{1} \xi_{0}\right] \\
& +\left(A \alpha_{1}+B u_{2 s}\right) \sinh \alpha_{1} \xi_{0} \cos \beta_{1} \xi_{0}-\left(A \beta_{1}-B \bar{u}_{2 s}\right) \cosh \alpha_{1} \xi_{0} \sin \beta_{1} \xi_{0} \\
k_{43}= & \frac{D}{R}\left[\left(\alpha_{2}^{2}-\beta_{2}^{2}\right) \sinh \alpha_{2} \xi_{0} \sin \beta_{2} \xi_{0}+2 \alpha_{2} \beta_{2} \cosh \alpha_{2} \xi_{0} \cos \beta_{2} \xi_{0}\right] \\
& +\left(A \alpha_{2}+B \bar{u}_{3 s}\right) \cosh \alpha_{2} \xi_{0} \sin \beta_{2} \xi_{0}+\left(A \beta_{2}+B u_{3 s}\right) \sinh \alpha_{2} \xi_{0} \cos \beta_{2} \xi_{0} \\
k_{44}= & \frac{D}{R}\left[\left(\alpha_{2}^{2}-\beta_{2}^{2}\right) \cosh \alpha_{2} \xi_{0} \cos \beta_{2} \xi_{0}-2 \alpha_{2} \beta_{2} \sinh \alpha_{2} \xi_{0} \sin \beta_{2} \xi_{0}\right] \\
& +\left(A \alpha_{2}+B u_{4 s}\right) \sinh \alpha_{2} \xi_{0} \cos \beta_{2} \xi_{0}-\left(A \beta_{2}-B \bar{u}_{4 s}\right) \cosh \alpha_{2} \xi_{0} \sin \beta_{2} \xi_{0} .
\end{aligned}
$$

Antisymmetric modes:

$$
\left(u_{1 a}, \bar{u}_{1 a}, u_{2 a}, \bar{u}_{2 a}, v_{1 a}, \bar{v}_{1 a}, v_{2 a}, \bar{v}_{2 a}\right)=\left(-u_{1 s}, \bar{u}_{1 s}, \bar{u}_{1 s}, u_{1 s}, v_{1 s},-\bar{v}_{1 s}, \bar{v}_{1 s}, v_{1 s}\right)
$$

The coefficients $\left(u_{3 a}, \bar{u}_{3 a}, v_{3 a}, \bar{v}_{3 a}\right)$ and $\left(u_{4 a}, \bar{u}_{4 a}, v_{4 a}, \bar{v}_{4 a}\right)$ remain the same as those of $\left(u_{1 a}, \bar{u}_{1 a}, v_{1 a}, \bar{v}_{1 a}\right)$ and $\left(u_{2 a}\right.$, $\left.\bar{u}_{2 a}, v_{2 a}, \bar{v}_{2 a}\right)$, respectively, with $\alpha_{1}$ and $\beta_{1}$ replaced by $\alpha_{2}$ and $\beta_{2}$.

$$
\begin{aligned}
& k_{1 i}=\left(\alpha_{1} u_{i a}-\beta_{1} \bar{u}_{i a}\right) \cosh \alpha_{1} \xi_{0} \sin \beta_{1} \xi_{0}+\left(\alpha_{1} \bar{u}_{i a}+\beta_{1} u_{i a}\right) \sinh \alpha_{1} \xi_{0} \cos \beta_{1} \xi_{0} \quad i=1,2 \\
& k_{1 i}=\left(\alpha_{2} u_{i a}-\beta_{2} \bar{u}_{i a}\right) \cosh \alpha_{2} \xi_{0} \sin \beta_{2} \xi_{0}+\left(\alpha_{2} \bar{u}_{i a}+\beta_{2} u_{i a}\right) \sinh \alpha_{2} \xi_{0} \cos \beta_{2} \xi_{0} \quad i=3,4 \\
& k_{2 i}=v_{i a} \sinh \alpha_{1} \xi_{0} \cos \beta_{1} \xi_{0}+\bar{v}_{i a} \cosh \alpha_{1} \xi_{0} \sin \beta_{1} \xi_{0} \quad i=1,2 \\
& k_{2 i}=v_{i a} \sinh \alpha_{2} \xi_{0} \cos \beta_{2} \xi_{0}+\bar{v}_{i a} \cosh \alpha_{2} \xi_{0} \sin \beta_{2} \xi_{0} \quad i=3,4 \\
& k_{31}=\sinh \alpha_{1} \xi_{0} \cos \beta_{1} \xi_{0} \quad k_{32}=\cosh \alpha_{1} \xi_{0} \sin \beta_{1} \xi_{0} \\
& k_{33}=\sinh \alpha_{2} \xi_{0} \cos \beta_{2} \xi_{0} \quad k_{34}=\cosh \alpha_{2} \xi_{0} \sin \beta_{2} \xi_{0} \\
& k_{41}=\frac{D}{R}\left[\left(\alpha_{1}^{2}-\beta_{1}^{2}\right) \sinh \alpha_{1} \xi_{0} \cos \beta_{1} \xi_{0}-2 \alpha_{1} \beta_{1} \cosh \alpha_{1} \xi_{0} \sin \beta_{1} \xi_{0}\right] \\
& +\left(A \alpha_{1}+B \bar{u}_{1 a}\right) \cosh \alpha_{1} \xi_{0} \cos \beta_{1} \xi_{0}-\left(A \beta_{1}-B u_{1 a}\right) \sinh \alpha_{1} \xi_{0} \sin \beta_{1} \xi_{0} \\
& k_{42}=\frac{D}{R}\left[\left(\alpha_{1}^{2}-\beta_{1}^{2}\right) \cosh \alpha_{1} \xi_{0} \sin \beta_{1} \xi_{0}+2 \alpha_{1} \beta_{1} \sinh \alpha_{1} \xi_{0} \cos \beta_{1} \xi_{0}\right] \\
& +\left(A \alpha_{1}+B u_{2 a}\right) \sinh \alpha_{1} \xi_{0} \sin \beta_{1} \xi_{0}+\left(A \beta_{1}+B \bar{u}_{2 a}\right) \cosh \alpha_{1} \xi_{0} \cos \beta_{1} \xi_{0} \\
& k_{43}=\frac{D}{R}\left[\left(\alpha_{2}^{2}-\beta_{2}^{2}\right) \sinh \alpha_{2} \xi_{0} \cos \beta_{2} \xi_{0}-2 \alpha_{2} \beta_{2} \cosh \alpha_{2} \xi_{0} \sin \beta_{2} \xi_{0}\right] \\
& +\left(A \alpha_{2}+B \bar{u}_{3 a}\right) \cosh \alpha_{2} \xi_{0} \cos \beta_{2} \xi_{0}-\left(A \beta_{2}-B u_{3 a}\right) \sinh \alpha_{2} \xi_{0} \sin \beta_{2} \xi_{0} \\
& k_{44}=\frac{D}{R}\left[\left(\alpha_{2}^{2}-\beta_{2}^{2}\right) \cosh \alpha_{2} \xi_{0} \sin \beta_{2} \xi_{0}+2 \alpha_{2} \beta_{2} \sinh \alpha_{2} \xi_{0} \cos \beta_{2} \xi_{0}\right] \\
& +\left(A \alpha_{2}+B u_{4 a}\right) \sinh \alpha_{2} \xi_{0} \sin \beta_{2} \xi_{0}+\left(A \beta_{2}+B \bar{u}_{4 a}\right) \cosh \alpha_{2} \xi_{0} \cos \beta_{2} \xi_{0} .
\end{aligned}
$$

